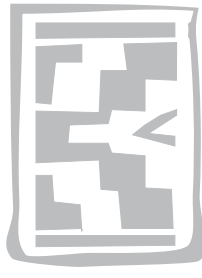

\title{
A check list of the helminths of guineafowls (Numididae) and a host list of these parasites
}

\author{
K. JUNKER and J. BOOMKER* \\ Department of Veterinary Tropical Diseases, Faculty of Veterinary Science, University of Pretoria \\ Private Bag X04, Onderstepoort, 0110 South Africa
}

\begin{abstract}
JUNKER, K. \& BOOMKER, J. 2007. A check list of the helminths of guineafowls (Numididae) and a host list of these parasites. Onderstepoort Journal of Veterinary Research, 74:315-337

Published and personal records have been compiled into a reference list of the helminth parasites of guineafowls. Where data on other avian hosts was available these have been included for completeness' sake and to give an indication of host range. The parasite list for the Helmeted guineafowls, Numida meleagris, includes five species of acanthocephalans, all belonging to a single genus, three trematodes belonging to three different genera, 34 cestodes representing 15 genera, and 35 nematodes belonging to 17 genera. The list for the Crested guineafowls, Guttera edouardi, contains a single acanthocephalan together with 10 cestode species belonging to seven genera, and three nematode species belonging to three different genera. Records for two cestode species from genera and two nematode species belonging to a single genus have been found for the guineafowl genus Acryllium. Of the 70 helminths listed for $N$. meleagris, 29 have been recorded from domestic chickens.
\end{abstract}

Keywords: Acanthocephalans, cestodes, check list, guineafowls, host list, nematodes, trematodes

\section{INTRODUCTION}

Guineafowls (Numididae) originated on the African continent, and with the exception of an isolated population of Helmeted guineafowls in north-west Morocco, their natural distribution is restricted to subSaharan Africa (Del Hoyo, Elliott \& Sargatal 1994). In the wake of commercial game bird farming, but also as ornamental birds in aviculture, they have been introduced to many other parts of the world, such as France, Hungary, Italy, Greece, the United Kingdom, the USA, Australia and different regions of the former USSR (Haziev \& Khan 1991). According to Belshaw (1985) guineafowls were imported

* Author to whom correspondence is to be directed. E-mail: joop.boomker@up.ac.za

Accepted for publication 9 May 2007-Editor into the southern Mediterranean region several millennia before turkeys and hundreds of years before junglefowls from which today's domestic chickens were derived. Currently four genera of guineafowls are recognized, namely Acryllium Gray, 1840, Agelastes Bonaparte, 1850, Guttera Wagler, 1832 and Numida Linnaeus, 1766 (Del Hoyo et al. 1994).

Many publications on the helminth fauna of guineafowls originate from northern and western Africa, where, second only to the introduced and native domestic fowls, they are farm-reared as a source of protein. The economic importance of guineafowls and domestic fowls within the poultry industry, as well as the fact that domestic fowls are kept by many private households to augment their income, necessitated a better understanding of factors, such as gastro-intestinal parasites, influencing the success- 
ful rearing of these birds. Consequently studies have been conducted to assess the extent to which guineafowls and domestic fowls can serve as alternative hosts for their respective helminths and possibly be adversely affected by them (Hodasi 1969, 1976; Fabiyi 1972; Fatunmbi \& Olufemi 1982; Vercruysse, Harris, Bray, Nagalo, Pangui \& Gibson 1985).

In southern Africa Ortlepp (1937, 1938a, b, 1963), Saayman (1966), Crowe (1977) and Verster \& Ptasinska-Kloryga (1987) have published on the helminth fauna of guineafowls. No data on the helminths infecting species of the guineafowl genus Agelastes could be found, and we are of the opinion that the comparatively short parasite lists for the genera Acryllium and Guttera reflect a lack of data rather than an absence of parasites.

The check list herein is intended as a quick reference aid and is split into two sections. The first section contains the parasites listed under their scientific names and authorities. Synonyms are provided either as generic synonyms in the case where whole genera have been synonymized or specific synonyms. The second section lists the hosts and their synonyms alphabetically, together with their respective parasites, also in alphabetical order.

The synonymy of the acanthocephalan genus Mediorhynchus Van Cleave 1916 is as given by Van Cleave (1947) and Schmidt \& Kuntz (1977) and specific synonymy is according to Yamaguti (1963). For an in-depth review of the involved history of this genus's nomenclature the reader is referred to Van Cleave (1947).

The taxonomy of digenean trematodes follows Yamaguti (1958), but since the application of molecular techniques to this group has recently led to many changes, the reader is encouraged to consult the latest literature.

The classification of cestodes is based on the works of Khalil, Jones \& Bray (1994). Information on generic synonyms and type species follows Khalil et al. (1994), while that on other species as well as the hosts and geographic distribution has mainly been derived from Yamaguti (1959), Schmidt (1986) and additional published records.

As regards nematode taxonomy, the authors have followed the ClH Keys to the nematode parasites of vertebrates (Anderson, Chabaud \& Willmott, 19741983) and, where differences have occurred, have accepted the validity of genera and species as listed by Gibson (2005). With regard to generic synonyms, only synonyms listed in the $\mathrm{ClH}$ keys and by Gibson
(2005) have been included in the check list. Specific synonyms, Type species and other species, as well as much of the data on hosts and geographic distribution are according to Yamaguti (1961) and Gibson (2005). Host and geographic data have been supplemented by including additional literature references.

The families and subfamilies of cestodes and nematodes are listed according to the system of Khalil et al. (1994) and the CIH Keys, respectively, but genera within these families are presented in alphabetical order. Synonyms have been arranged chronologically. The hosts and geographic localities per author are listed alphabetically. If several authors made reference to the same host, the authors are listed in chronological order.

The nomenclature and taxonomy of the avian hosts mainly follows Peterson (1999) and has been supplemented by Lepage (2007). Avian orders and families, as well as the nomenclature of southern African hosts follow Hockey, Dean \& Ryan (2005).

In order to avoid excessive duplication, Helmeted guineafowls are listed below as $N$. meleagris only without regards to the subspecies. A total of nine subspecies of $N$. meleagris are currently recognized (Del Hoyo et al. 1994, Peterson 1999). These are: N. m. coronatus Gurney, 1868, N. m. galeatus Pallas, $1767, N$. m. marungensis Schalow, 1884, N. m. meleagris (Linnaeus, 1758), N. m. mitratus Pallas, 1767, N. m. papillosus Reichenow, 1894, N. m. reichenowi Ogilvie-Grant, 1894, N. m. sabyi Hartert, 1919 and N. m. somaliensis Neumann, 1899. Del Hoyo et al. (1994) give a detailed list of the geographic range of the various subspecies of Helmeted guineafowls.

In the case of the Crested guineafowls, Guttera edouardi (Hartlaub, 1867) we follow Hockey et al. (2005) and Lepage (2007). Crowe (1978, cited in Hockey et al. 2005) had synonymized G. edouardi with Guttera pucherani (Hartlaub, 1861), but this decision was reversed and G. edouardi reinstated (Little \& Crowe 2000, cited in Hockey et al. 2005). Peterson (1999) still lists $G$. edouardi as a subspecies of $G$. pucherani.

Hosts listed in the literature as Gallus domesticus or Gallus gallus domesticus are referred to below as domestic chicken. Lepage (2007) lists domestic chicken as unconfirmed subspecies, G. g. domesticus (no authority given), of the Red Junglefowl, Gallus gallus (Linnaeus, 1758). However, this subspecies is not included in the five subspecies listed by Peterson (1999). 


\section{PARASITE/HOST CHECK LIST}

PHYLUM ACANTHOCEPHALA

\section{Class Archiacanthocephala}

Order Gigantorhynchidea

Family GIGANTORHYNCHIDAE Hamann, 1892

Genus Mediorhynchus Van Cleave 1916

Echinorhynchus Zoega in Müller, 1776, in part; Gigantorhynchus Hamann,1892, in part; Heteroplus Kostylev, 1914; Empodius Travassos, 1916; Micracanthorhynchus Travassos, 1917; Leiperacanthus Bhalerao, 1937; Disteganius Lehmann, 1953, nomen nudum; Empodisma Yamaguti, 1963

Type species: Mediorhynchus papillosus Van Cleave, 1918

1. Mediorhynchus empodius (Skrjabin, 1913) Meyer, 1933

Ardea, Ardeotis arabs, Numida meleagris Yamaguti (1963), Belgium, Russia

2. Mediorhynchus gallinarum (Bhalerao, 1937) Van Cleave, 1947

Domestic chicken Yamaguti (1963), India, Philippines Talbot (1971), Papua and New Guinea

Gallinaceaous birds Schmidt \& Kuntz (1977), (East-) Africa, India, Papua and New Guinea, Philippines

Numida meleagris Junker \& Boomker (2006), South Africa

3. Mediorhynchus numidae (Baer, 1925) Meyer, 1933

Numida meleagris Meyer (1932), Namibia

Oosthuizen \& Markus (1967), South Africa

4. Mediorhynchus selengensis Harris, 1973

Numida meleagris

Vercryusse et al. (1985), Burkina Faso

Schmidt \& Kuntz (1977) synonymized this species with M. gallinarum.

5. Mediorhynchus taeniatus (Von Linstow, 1901) Dollfus, 1936

Empodius segmentatus De Marval, 1902

Ardeotis arabs

Dollfus (1951) in Yamaguti (1963), Mauritania

Chlamydotis undulata

Dollfus (1951) in Yamaguti (1963), Morocco

Guttera edouardi

Southwell \& Lake (1939), Democratic Republic of the Congo

Numida meleagris

Von Linstow (1901), Kenya

Meyer (1932), Africa, Malawi

Southwell \& Lake (1939), Democratic Republic of the Congo

Graber (1959), Chad
Fabiyi (1972), Nigeria

Hodasi (1976), Ghana

Crowe (1977), South Africa

Burhinus oedicnemus, Chlamydotis macquenii, Otis tarda

Meyer (1932), Africa, Malawi

PHYLUM PLATHYHELMINTHES

\section{Class Trematoda}

\section{Order Digenea}

Family BRACHYLAEMIDAE Joyeaux \& Foley, 1930

Genus Postharmostomum Witenberg, 1923

Type species: Postharmostomum gallinum (Witenberg, 1923)

1. Postharmostomum gallinum (Witenberg, 1923)

Crested guineafowl Khan, Khan \& Rayaz (1984), Pakistan

Domestic chicken Yamaguti (1958), Hawaii, Japan, Russian Turkestan

Numida meleagris

Yamaguti (1958), North Africa

\section{Family DICROCOELIIDAE Odhner, 1911}

\section{Genus Dicrocoelium Dujardin, 1845}

Type species: Dicrocoelium lanceatum Stiles \& Hassal, 1898

1. Dicrocoelium macrostomum Odhner, 1911

Coturnix coturnix

Yamaguti (1958), Russia

Numida meleagris

Lesbouyries (1941), Egypt

Junker \& Boomker (2007b), South Africa

Genus Lutztrema travassos, 1941

Type species: Lutztrema olliquum (Travassos, 1917)

Numida meleagris

Hodasi (1976), Ghana

\section{Class Cestoda}

\section{Subclass Eucestoda}

Order Cyclophyllidea

Family DAVAINEIDAE Braun, 1900

Subfamily Davaineinae Braun, 1900

Genus Abuladzugnia Spasskil, 1973

Type species: Abuladzugnia gutterae (Ortlepp, 1963)

1. Abuladzugnia gutterae (Ortlepp, 1963)

Cotugnia gutterae Ortlepp, 1963

Guttera edouardi

Ortlepp (1963), Mozambique

Numida meleagris

Junker \& Boomker (2007b), South Africa 
Check list of helminths of guineafowls (Numididae) and host list of parasites

2. Abuladzugnia transvaalensis (Ortlepp, 1963)

Cotugnia transvaalensis Ortlepp, 1963

Numida meleagris

Ortlepp (1963), South Africa

\section{Genus Cotugnia Diamare, 1893}

Ershovitugnia, Spasskii, 1973; Pavugnia Spasskii, 1984; RosteIugnia Spasskii, 1984

Type species: Cotugnia digonopora (Pasquale, 1890) Diamare, 1893

1. Cotugnia crassa Fuhrmann, 1909

Guineafowl

Hudson (1934), East Africa

Bwangamoi (1968), Uganda

Numida meleagris

Fuhrmann (1909) in Ortlepp (1963), the White Nile

Baer (1925), Namibia

Baer (1926), East Africa, West Africa

Ortlepp (1963), Tanzania

The White Nile rises from Lake Victoria in Uganda and enters the Sudan where it joins the Blue Nile in Karthoum to form the Nile. White Nile is one of the states of Sudan.

2. Cotugnia digonopora (Pasquale, 1890) Diamare, 1893 Taenia digonopora Pasquale, 1890

Anser, Columba livia, Gallus gallus, Numida meleagris Schmidt (1986), Africa, Burma, India, Indonesia, Philippines

Guineafowl

Baylis (1934), Uganda

3. Cotugnia meleagridis Joyeux, Baer \& Martin, 1936

Numida meleagris

Joyeux, Baer \& Martin (1936), Northern Somaliland

Graber (1959), Chad

Fabiyi (1972), Nigeria

Hodasi (1976), Ghana

4. Cotugnia shohoi Sawada, 1971

Acryllium vulturinum

Schmidt (1986), Somalia

5. Cotugnia tuliensis Mettrick, 1963

Numida meleagris

Schmidt (1986), Zimbabwe

\section{Genus Davainea Blanchard, 1891}

Type species: Davainea proglottina (Davaine, 1860) Blanchard, 1891

1. Davainea nana Fuhrmann, 1912

Guttera edouardi

Ortlepp (1963), Zambia

Numida meleagris

Fuhrmann (1912), Northern Africa

Junker \& Boomker (2007b), South Africa

Vanellus cinereus

Schmidt (1986), Africa, Japan
2. Davainea paucisegmentata Fuhrmann, 1909

Numida meleagris

Baer (1926), Sudan, West Africa

Schmidt (1986), Africa, Europe

3. Davainea paucisegmentata var. dahomeensis Joyeux \& Baer, 1928

Numida meleagris

Schmidt (1986), France

4. Davainea proglottina (Davaine, 1860) Blanchard, 1891

Taenia proglottina Davaine, 1860; Davainea varians Sweet, 1910; Davainea dubius Meggitt, 1916

Alectoris graeca, Bonasa umbellus, Gallus gallus, Perdix perdix

Schmidt (1986), Cosmopolitan

Domestic chicken

Baer (1926), South Africa

Magwisha, Kassuku, Kyvsgaard \& Permin (2002),

Tanzania

Numida meleagris

Nfor, Ajanusi, Agbede \& Esievo (1999), Nigeria

\section{Genus Numidella Spasskaya \& Spasskil, 1971}

Type species: Numidella numida (Fuhrmann, 1912) Spasskaya \& Spasskii, 1971

1. Numidella numida (Fuhrmann, 1912) Spasskaya \& Spasskii, 1971

Davainea numida Fuhrmann, 1912; Raillietina (Paroniella) numida (Fuhrmann, 1912) Fuhrmann, 1920; Raillietina (Paroniella) magninumida Jones, 1930

Guineafowl

Baylis (1934), Uganda

Guttera, Numida meleagris

Schmidt (1986), Africa, Cuba, North America

Guttera

Baer (1933), Zimbabwe

Baer (1933) lists 'Guttera eduardi Elliot' as host. None of the subspecies of Guttera edouardi listed in Lepage (2007) has been described by Elliot, but Lepage (2007) lists Guttera pucherani verreauxi (Elliot, 1870).

Meleagris gallopavo, Numida meleagris Jones (1930), North America

Numida meleagris

Baer (1925), Namibia

Ortlepp (1963), South Africa

Fabiyi (1972), Nigeria

\section{Genus Porogynia Railliet \& Henry, 1909}

Polycoelia Fuhrmann, 1907, preoccupied

Type species: Porogynia paronai (Moniez, 1892) Railliet \& Henry, 1909

1. Porogynia paronai (Moniez, 1892) Railliet \& Henry, 1909

Taenia paronai Moniez, 1892; Linstowia lata Fuhrmann, 1901; Polycoelia lata (Fuhrmann, 1901) Fuhrmann, 1907; Malika numida Woodland, 1929; Raillietina (Paroniella) woodlandi Baylis, 1934 
Guttera edouardi, Numida meleagris, Pternistis natalensis

Schmidt (1986), Africa, Europe

Guineafowl

Woodland (1928), Sudan

Baylis (1934), Uganda

Guttera edouardi

Ortlepp (1963), Zambia

Numida meleagris

Baer (1925), Namibia

Baer (1926), East Africa, West Africa

Woodland (1928), Sudan

Ortlepp (1963), South Africa, Swaziland

Cruz e Silva (1971), Mozambique

Fabiyi (1972), Nigeria

\section{Genus Raillietina Fuhrmann, 1920}

Kotlania López-Neyra, 1929; Nonarmiella Movsesyan, 1966; Nonarmina Movsesyan, 1966; Kotlanotaurus Spasskii, 1973; Roytmania Spasskii, 1973; Skrjabinotaurus Spasskii \& Yurpalova, 1973; Oschmarinetta Spasskii, 1984

Type species: Raillietina tetragona (Molin, 1858)

1. Raillietina angusta Ortlepp, 1963

Raillietina (Raillietina) angusta Ortlepp, 1963

Numida meleagris

Ortlepp (1963), South Africa

2. Raillietina cohni (Baczynska, 1914) Fuhrmann, 1924

Davainea cohni Baczynska, 1914; Raillietina (Ransomia) cohni (Baczynska, 1914) Fuhrmann, 1920; Raillietina (Raillietina) cohni (Baczynska, 1914) Fuhrmann, 1924

Gallus gallus, Numida meleagris, Pterocles exustus, Pterocles orientalis arenarius

Schmidt (1986), Africa, Nepal

Domestic chicken

Baer (1926), East Africa

3. Raillietina echinobothrida (Megnin, 1880) Fuhrmann, 1924

Taenia echinobothrida Megnin, 1880; Taenia botrioplites Piana, 1881; Davainea parechinobothrida Magalhães, 1898; Davainea penetrans Baczynska, 1914; Raillietina (Johnstonia) echinobothrida (Megnin, 1880) Fuhrmann, 1920; Raillietina (Raillietina) echinobothrida (Megnin, 1880) Fuhrmann, 1924; Raillietina (Fuhrmannetta) echinobothrida (Megnin, 1880) Stiles \& Orleman, 1926

Columba livia, Gallus gallus, Gallus gallus bankiva, Meleagris gallopavo, Numida meleagris, Perdix perdix, Phasianus colchicus

Schmidt (1986), Cosmopolitan

Domestic chicken

Baer (1926), West Africa

Le Roux (1926), South Africa

Joyeux et al. (1936), Northern Somalia

Poulsen, Permin, Hindsbo, Yelifari, Nansen \& Bloch (2000), Ghana

Magwisha et al. (2002), Tanzania

Permin, Esmann, Hoj, Hove \& Mukaratirwa (2002),

Zimbabwe
Gallus gallus bankiva Baer (1933), Zimbabwe

Numida meleagris Baer (1933), Zimbabwe

Southwell \& Lake (1939), Democratic Republic of the Congo

Cruz e Silva (1971), Mozambique

Ayeni, Dipeolu \& Okaeme (1983), Nigeria

4. Raillietina pintneri (Klaptocz, 1906) Fuhrmann, 1924

Davainea pintneri Klaptocz, 1906; Raillietina (Ransomia) pintneri (Klaptocz, 1906) Fuhrmann, 1920; Raillietina (Raillietina) pintneri (Klaptocz, 1906) Fuhrmann 1924; Kotlania pintneri (Klaptocz, 1906) López-Neyra, 1931

Guttera

Baer (1933), Zimbabwe

Baer (1933) lists 'Guttera eduardi Elliot' as host. None of the subspecies of Guttera edouardi listed in Lepage (2007) have been described by Elliot, but Lepage (2007) lists Guttera pucherani verreauxi (Elliot, 1870).

Guttera edouardi, Numida meleagris Schmidt (1986), Africa

Guttera edouardi Ortlepp (1963), Mozambique, Zambia

Numida meleagris

Baer (1925), Namibia

Baer (1926), Sudan, West Africa

Graber (1959), Chad

Ortlepp (1963) South Africa, Swaziland

Fabiyi (1972), Nigeria

5. Raillietina somalensis Sawada, 1971

Raillietina (Raillietina) somalensis Sawada, 1971

Acryllium vulturinum

Schmidt (1986), Somalia

6. Raillietina steinhardti Baer, 1925

Raillietina (Ransomia) steinhardti Baer, 1925

Guttera edouardi

Ortlepp (1963), Mozambique, Zambia

Numida meleagris

Yamaguti (1959), Africa

Verster \& Ptasinska-Kloryga (1987), South Africa

7. Raillietina tetragona (Molin, 1858) Fuhrmann, 1924

Taenia tetragona Molin, 1858; Taenia Iongicollis Molin, 1858; Davainea tetragona (Molin, 1858) Blanchard, 1891; Davainea bothrioplitis Fillippi, 1892; Raillietina (Ransomia) tetragona (Molin, 1858) Fuhrmann, 1920; Raillietina (Raillietina) tetragona (Molin, 1858) Fuhrmann, 1924; Kotlania tetragona (Molin, 1858) López-Neyra, 1931; Raillietina (Raillietina) galli (Yamaguti, 1935) Sawada, 1955

Gallus gallus, Guttera edouardi, Lagopus lagopus, Lagopus muta, Meleagris gallopavo, Numida meleagris, Pavo cristatus, Pavo muticus

Schmidt (1986), Cosmopolitan

Domestic chicken

Baer (1926), East Africa, West Africa

Le Roux (1926), South Africa

Poulsen et al. (2000), Ghana 
Magwisha et al. (2002), Tanzania

Permin et al. (2002), Zimbabwe

Numida meleagris

Baer (1926), East Africa, West Africa

Ayeni et al. (1983), Nigeria

Haziev \& Khan (1991), Republic of Bashkortostan

8. Raillietina tetragonoides (Baer, 1925) Fuhrmann, 1932 Raillietina (Ransomia) tetragonoides Baer, 1925; Raillietina (Raillietina) tetragonoides (Baer, 1925) Fuhrmann, 1932; Raillietina (Raillietina) tetragona var. cohni (Baczynska, 1914) López-Neyra, 1944

Numida meleagris

Baer (1925), Namibia

Schmidt (1986), Africa

9. Raillietina toyohashiensis Sawada \& Chikada, 1972

Numida meleagris

Schmidt (1986), Japan (zoo)

\section{Genus SkRJabinia Fuhrmann, 1920}

Raillietina (Skrjabinia) Fuhrmann, 1920; Brumptiella López-Neyra, 1929; Armacetabulum Movsesyan, 1966; Markewitchella Spasskii \& Spasskaya, 1972; Daovantienia Spasskii \& Spasskaya, 1976

Type species: Skrjabinia cesticillus (Molin, 1858) Fuhrmann, 1920

1. Skrjabinia cesticillus (Molin, 1858) Fuhrmann, 1920

Taenia cesticillus Molin, 1858; Davainea cesticillus Blanchard, 1891; Raillietina (Raillietina) mutabilis Rüther, 1901; Raillietina (Skrjabinia) cesticillus (Molin, 1858) Fuhrmann, 1920

Colinus virginianus, Coturnix coturnix, Gallus gallus, Lagopus lagopus, Lagopus lagopus scotica, Lyrurus tetrix, Meleagris gallopavo, Numida meleagris, Perdix perdix, Phasianus colchicus, Tetrao urogallus, Tetrastes bonasia

Schmidt (1986), Cosmopolitan

Domestic chicken

Baer (1926), West Africa

Le Roux (1926), South Africa

Joyeux et al. (1936), Northern Somalia

Poulsen et al. (2000), Ghana

Magwisha et al. (2002), Tanzania

Permin et al. (2002), Zimbabwe

Numida meleagris

Nfor et al. (1999), Nigeria

2. Skrjabinia deweti Ortlepp, 1938

Numida meleagris

Ortlepp (1938a), South Africa

\section{Subfamily Idiogeninae Fuhrmann, 1907}

\section{Genus IDIOGenes KrabBe, 1867}

Ersinogenes Spasskaya, 1961; Paraidiogenes Movsesyan, 1971

Type species: Idiogenes otidis Krabbe, 1867

1. Idiogenes sp.

Numida meleagris

Hodasi (1976), Ghana

\section{Family DILEPIDIDAE Railliet \& Henry, 1909}

\section{Genus Choanotaenia Railliet, 1896}

Type species: Choanotaenia infundibulum (Bloch, 1779) Railliet, 1896

1. Choanotaenia infundibulum (Bloch, 1779) Railliet, 1896

Taenia infundibulum Bloch, 1779

Domestic chicken

Poulsen et al. (2000), Ghana

Magwisha et al. (2002), Tanzania

Numida meleagris

Haziev \& Khan (1991), Republic of Bashkortostan

Nfor et al. (1999), Nigeria

\section{Family PARUTERINIDAE Fuhrmann, 1907}

\section{Genus Octopetalum Baylis, 1914}

Type species: Octopetalum gutterae Baylis, 1914

1. Octopetalum gutterae Baylis, 1914

Ascometra gutterae (Baylis, 1914) Baer,1955

Guttera edouardi, Numida meleagris

Baer (1926), East Africa

Baer (1955), Democratic Republic of Congo, Malawi, South Africa

Schmidt (1986) Africa, France

2. Octopetalum numida (Fuhrmann, 1909) Baylis, 1914 Rhabdometra numida Fuhrmann, 1909; Octopetalum longicirrosum Baer, 1925; Unciunia sudanea Woodland, 1928; Ascometra numida (Fuhrmann, 1909) Baer, 1955

Guineafowl Baylis (1934), Uganda

Guttera edouardi

Baer (1955), Sub-Saharan Africa

Ortlepp (1963), South Africa, Zambia

Numida meleagris

Baer (1925), Namibia

Baer (1926), Sudan, West Africa

Woodland (1928), Sudan

Baer (1955), Sub-Saharan Africa

Ortlepp (1963), Central Africa, North Africa, South

Africa, southern Africa, Swaziland

Fabiyi (1972), Nigeria

Hodasi (1976), Ghana

\section{Genus Metroliasthes Ransom, 1900}

Hexaparuterina Palacios \& Barroeta, 1967

Type species: Metroliasthes Iucida Ransom, 1900

1. Metroliasthes lucida Ransom, 1900

Alectoris graeca, Alectoris rufa, Coturnix coturnix, Gallus gallus, Gallus gallus bankiva, Guttera edouardi, Meleagris gallopavo, Numida meleagris, Perdix perdix Schmidt (1986), Africa, Australia, Europe, India, North and South America, Russia

Numida meleagris

Southwell \& Lake (1939), Democratic Republic of Congo 


\section{Family HYMENOLEPIDIDAE Ariola, 1899}

\section{Subfamily Hymenolepidinae Perrier, 1897}

\section{Genus EChINOLEPIS SPASSKII \& SPASSKAyA, 1954}

Type species: Echinolepis carioca (Maghalães, 1898) Spasskii \& Spasskaya, 1954

1. Echinolepis carioca (Maghalães, 1898) Spasskii \& Spasskaya, 1954

Davainea carioca Maghalães, 1898; Taenia conardi Zürn, 1898; Hymenolepis carioca (Maghalães, 1898) Ransom, 1902; Hymenolepis pullae Cholodkovsky, 1913; Weinlandia rustica Meggitt, 1926; Hymenolepis rustica Fuhrmann, 1932; Dicranotaenia carioca (Maghalães, 1898) Skrjabin \& Mathevossian, 1945; Dicranotaenia rustica (Meggitt, 1926) Skrjabin \& Mathevossian, 1945

Alectoris graeca, Bonasa umbellus, Colinus virginianus, Coturnix coturnix, Gallus gallus, Meleagris gallopavo

Schmidt (1986), Cosmopolitan

Domestic chicken

Le Roux (1926), South Africa

Magwisha et al. (2002), Tanzania

Numida meleagris

Baer (1926), West Africa

\section{Genus Hymenolepis WeinLand, 1858}

Triorchis Clerc, 1903 preoccupied; Cloacotaenia Wolffhügel, 1938; Amphipetrovia Spasskii \& Spasskaya, 1954; Australiolepis Spasskii \& Spasskaya, 1954; Orlovilepis Spasskii \& Spasskaya, 1954; Staphylepis Spasskii \& Oshmarin, 1954; Arhynchotaenia Saakova, 1958 nec Pagenstecher, 1877; Schmelzia Yamaguti, 1959; Woodlandia Yamaguti, 1959; Arhynchotaeniella Schmidt, 1986; Cloacotaeniella Schmidt, Bauerle \& Wertheim, 1988; Amazilolepis Schmidt \& Daily, 1992

Type species: Hymenolepis diminuta (Rudolphi, 1819) Weinland, 1858

1. Hymenolepis cantaniana (Polonio, 1860) Ransom, 1909

Taenia cantaniana Polonio, 1860; Davainea oligophora Maghalães, 1898; Davainea cantaniana Railliet \& Lucet, 1899; Hymenolepis inermis (Yoshida, 1910) Fuhrmann, 1932

Colinus virginianus, Coturnix coturnix, Gallus gallus, Meleagris gallopavo, Numida meleagris, Pavo cristatus, Perdix perdix, Phasianus colchicus, Tetrao parvirostris, Tetrastes bonasia, Turnix suscitator

Schmidt (1986), Cosmopolitan

Domestic chicken

Le Roux (1926), South Africa

Magwisha et al. (2002), Tanzania

Le Roux (1926) chose to retain the name $H$. inermis for his unarmed specimens and not to accept the synonymy of $H$. inermis and $H$. cantaniana since the latter had been described as having an armed rostellum.

Numida meleagris

Hodasi (1976), Ghana

Junker \& Boomker (2007b), South Africa

\section{Genus Hispaniolepis Lòpez-NeYra, 1942}

Satyolepis Spasskii, 1965

Type species: Hispaniolepis villosa (Bloch, 1782) López-Neyra, 1942

1. Hispaniolepis falsata (Meggitt, 1927) López-Neyra, 1942

Hymenolepis falsata Meggitt, 1927.

Numida meleagris

Myers, Wolfgang \& Kuntz (1960), Sudan

Chlamydotis undulata

Schmidt (1986), Egypt

2. Hispaniolepis fedtschenkoi (Solowiow, 1911) LópezNeyra, 1942

Hymenolepis fedtschenkoiSolowiow, 1911; Hymenolepis gwiletica Dinnik, 1938

Gallus gallus, Lyrurus tetrix, Numida meleagris, Tetraogallus himalayensis, Tetraogallus caucasicus, Tetrastes bonasia

Schmidt (1986), Russia, Europe, Asia, Africa

3. Hispaniolepis hilmyi (Skrjabin \& Mathevossian, 1942) López-Neyra, 1942

Hymenolepis tetracis Hilmy, 1936

Numida meleagris

Schmidt (1986), Liberia

4. Hispaniolepis villosa (Bloch, 1782) López-Neyra, 1942 Numida meleagris

Baer (1926), East Africa

\section{Genus Ortleppolepis Spasskil, 1965}

Type species: Ortleppolepis multiuncinata (Ortlepp, 1963) Spasskii, 1965

1. Ortleppolepis multiuncinata (Ortlepp, 1963) Spasskii, 1965

Hispaniolepis multiuncinata Ortlepp, 1963

Guttera edouardi

Ortlepp (1963), Zambia

Numida meleagris

Junker \& Boomker (2007b), South Africa

PHYLUM NEMATHELMINTHES

\section{Class Nematoda}

Subclass Adenophorea

Order Enoplida

Superfamily Trichinelloidea Hall, 1916

Family TRICHURIDAE (Ransom, 1911) Railliet, 1915

Subfamily Capillariinae Railliet, 1915

Genus AonchothecA López-neyrA, 1947

Avesaonchotheca auct.; Baruscapillaria auct.; Capillaria auct.; Pterothomix auct.; Skrjabinocapillaria Skarbilovich, 1946

1. Aonchotheca caudinflata (Molin, 1858) 
Calodium caudinflata Molin, 1858; Capillaria blomei Travassos, 1915; Trichosoma longicollis Rudolphi, 1819

Chrysolophus, Columba, Coturnix, Gallus, Lagopus, Lyrurus, Otis, Numida meleagris, Passer, Perdix, Phasianus, Sturnus, Tetrao, Turdus

Yamaguti (1961), Europe, North America

Yamaguti (1961) lists Aonchotheca caudinflata from Otis without giving the host's species name. It is therefore not clear whether Otis refers to the current genus Otis, Ardeotis, Neotis or Chlamydotis.

Domestic chicken

Magwisha et al. (2002), Tanzania

Numida meleagris

Ayeni et al. (1983), Nigeria

\section{Genus Capillaria Zeder, 1800}

Trichosoma Rudolphi, 1819; Trichosomum Creplin, 1829; Thominx Dujardin, 1845; Tridentocapillaria Barus \& Sergeeva, 1990; Aonchotheca auct; Baruscapillaria auct.; Ptherominxauct.; Trichocephalus auct.

Type species: Capillaria anatis (Schrank, 1790) Travassos, 1915

1. Capillaria anatis (Schrank, 1790)

Trichocephalus capillaris Rudolphi, 1809

Anas, Anser, Clangula, Lyrurus, Melanitta, Merganser, Perdix, Phasianus

Yamaguti (1961), Europe, Sakhalin, Siberia

Domestic chicken

Magwisha et al. (2002), Tanzania

Numida meleagris

Nfor et al. (1999), Nigeria

\section{GENUS EuColeus DuJARDIN, 1845}

Capillaria auct.; Thominx auct.; Trichocephalus auct.

1. Eucoleus annulatus (Molin, 1858)

Trichosoma annulatus Molin, 1858

Bonasa, Chrysolophus, Colinus, Gallus, Lyrurus, Meleagris, Numida meleagris, Perdix, Phasianus, Syrmaticus, Tetrao

Yamaguti (1961), Asia, Europe, North and South America

Domestic chicken

Magwisha et al. (2002), Tanzania

Numida meleagris

Fabiyi (1972), Nigeria

Hodasi (1976), Ghana

Vercruysse et al. (1985), Burkina Faso

\section{Subclass Secernentea}

\section{Order Rhabditida}

\section{Superfamily Rhabditoidea}

\section{Family STRONGYLOIDIDAE Chitwood \& McIntosh,} 1934

\section{Genus Strongyloides Grassi, 1879}

1. Strongyloides avium Cram, 1929
Gallus

Yamaguti (1961), North America, Puerto Rico

Numida meleagris

Fabiyi (1972), Nigeria

\section{Order Strongylida}

Superfamily Strongyloidea

\section{Family SYNGAMIDAE Leiper, 1912}

Subfamily Syngaminae Baylis \& Daubney, 1926

Genus Syngamus Siebold, 1836

Cyathostoma auct.; Ornithogamus Ryjikov, 1948

Type species: Syngamus trachea (Montagu, 1811) Siebold, 1836

1. Syngamus trachea (Montagu, 1811) Siebold, 1836

Fasciola trachea Montagu, 1811; Syngamus trachealis Siebold, 1836; Strongylus trachealis Nathusius, 1937 in Ortlepp (1923); Strongylus pictus Creplin, 1849; Sclerostomum syngamus Diesing, 1951 in Ortlepp (1923); Syngamus furcatus Theob., 1896; Syngamus primitivus Molin, 1861; Syngamus sclerostomum Molin, 1861

Galliformes, Passeriformes; rarely Anseriformes, "Ardeiformes", "Pelicaniformes", Piciformes, Otidiformes Yamaguti (1961), Africa, Australia, Europe, India, North and South America

Domestic chicken Magwisha et al. (2002), Tanzania

Numida meleagris

Hodasi (1976), Ghana

Nfor et al. (1999), Nigeria

\section{Order Ascaridida}

Superfamily Heterakoidea

\section{Family HETERAKIDAE Railliet \& Henry, 1912}

Subfamily Heterakinae Railliet \& Henry, 1912

\section{Genus Heterakis DuJARdin, 1845}

Ganguleterakis Lane, 1914; Raillietakis Freitas, 1956; Inglisakis Freitas, Vicente \& Santos, 1969

Type species: Heterakis vesicularis (Frölich, 1791)

1. Heterakis vesicularis (Frölich, 1791)

Ascaris vesicularis Frölich, 1791; Ascaris papillosa, Bloch, 1782, in part

Anas, Colinus, Coturnix, Cygnus, Gallus, Lagopus, Meleagris, Numida meleagris, Oreortyx pictus, Otis, Pavo, Perdix, Phasianus colchicus, Polyplectron, Tetrao Yamaguti (1961), Africa, Europe, North America

Lophophorus, Lophura

Yamaguti (1961), Nepal

Yamaguti (1961) lists Heterakis vesicularis from Otis without giving the host's species name. It is therefore not clear whether Otis refers to the current genus Otis, Ardeotis, Neotis or Chlamydotis.

2. Heterakis brevispiculum Gendre, 1911

Domestic chicken, Numida meleagris, Pternistis bicalcaratus 
Yamaguti (1961), Africa, Puerto Rico, South America

Numida meleagris

Fabiyi (1972), Nigeria

Hodasi (1976), Ghana

3. Heterakis dispar (Schrank, 1790)

Ascaris dispar Schrank, 1790

Alectoris, Anas, Anser, Anser cygnoides, Branta, Bernicla, Cairina, Chloephaga, Glaucidium, Numida meleagris, Strix, Surnia, Tadorna

Yamaguti (1961), Cosmopolitan

Domestic chicken

Permin, Magwisha, Kassuku, Nansen, Bisgaard, Frandsen \& Gibbons (1997), Tanzania

4. Heterakis gallinarum (Schrank, 1788)

Ascaris gallinarum Schrank, 1788; Heterakis gallinae Gmelin, 1790; Heterakis longicaudata Von Linstow, 1879

Acryllium, Alectoris, Anas, Anser, Bonasa, Cairina, Chrysolophus, Colinus, Corvus, Coturnix, Cupidonia, domestic chicken, Francolinus, Houbara, Lagopus, Lophophorus, Lophura, Lyrurus, Meleagris, Otis, Pavo, Pedioecetes, Perdix, Phasianus, Pterocles, Strix, Syrmaticus, Tetrao, Tragopan, Tympanuchus

Yamaguti (1961), Cosmopolitan

Yamaguti (1961) lists Heterakis gallinarum from Otis without giving the host's species name. It is therefore not clear whether Otis refers to the current genus Otis, Ardeotis, Neotis or Chlamydotis.

Domestic chicken

Poulsen et al. (2000), Ghana

Magwisha et al. (2002), Tanzania

Permin et al. (2002), Zimbabwe

Numida meleagris

Ayeni et al. (1983), Nigeria

Verster \& Ptasinska-Kloryga (1987), South Africa Haziev \& Khan (1991), Republic of Bashkortostan Santa Cruz, Ortis de Rott \& Resoagli (1998), Argentina

5. Heterakis tenuicauda Von Linstow, 1883

Alectoris graeca, Alectoris graeca saxatilis

Yamaguti (1961), Turkestan

Acryllium vulturinum

Canavan (1929) in Yamaguti (1961), East Africa

\section{Family ASCARIDIIDAE Travassos, 1919}

\section{Genus Ascaridia DUJARdin, 1845}

Cotylascaris Sprent, 1971

Type species: Ascaridia hermaphrodita (Frölich, 1789) Railliet \& Henry, 1914

1. Ascaridia calcarata (Gendre, 1909)

Numida meleagris

Yamaguti (1961), Africa

Junior synonym of Ascaridia numidae (Leiper, 1908) according to Sprehn (1932) in Yamaguti (1961).

2. Ascaridia compar (Schrank, 1790) Travassos, 1913
Ascaris compar Schrank, 1790

Alectoris, Coturnix, Gallus, Lyrurus, Numida meleagris, Oreortyx pictus, Perdix, Tetrao, Tetrastes

Yamaguti (1961), America, Europe, India, Philippines

3. Ascaridia galli (Schrank, 1788) Freeborn, 1932

Ascaris galli Schrank, 1788; Fusaria inflexa Zeder, 1800 (Baylis 1932, cited in Yamaguti 1961); Fusaria reflexa Zeder, 1800, in part; Fusaria strumosa Zeder, 1800, in part (López-Neyra 1946, cited in Yamaguti 1961); Heterakis brasiliensis Magalhães, 1892 (Pinto \& Lins de Almeida 1935, cited in Yamaguti 1961); Heterakis granulosa Von Linstow, 1906 (Baylis 1932, cited in Yamaguti 1961); Ascaridia hamia Lane, 1914

Domestic chicken, guineafowl Yamaguti (1961), Europe, Japan

Alectoris, Bonasa, Cairina, Colinus, duck, Ithaginis, Lyrurus, Meleagris, Numida meleagris, Perdix, Phasianus, Streptopelia, Tetrao, Tympanuchus

Yamaguti (1961), Cosmopolitan

Domestic chicken

Poulsen et al. (2000), Ghana

Magwisha et al. (2002), Tanzania

Permin et al. (2002), Zimbabwe

Numida meleagris

Ayeni et al. (1983), Nigeria

Verster \& Ptasinska-Kloryga (1987), South Africa

Haziev \& Khan (1991), Republic of Bashkortostan

4. Ascaridia lineata (Schrank, 1866)

Ascaris lineata Schrank, 1866

Alectoris, Anas, Anser, Bonasa, duck, Francolinus, Gallus, goose, Meleagris, Meleagris ocellata, Numida, partridge, Phasianus, pigeon, Tympanuchus

Yamaguti (1961), Africa, Brazil, China, Cuba, Europe, Formosa, India, Malaya, North America, Philippines, Puerto Rico, Turkestan

Domestic chicken

Le Roux (1926), South Africa

5. Ascaridia numidae (Leiper, 1908) Travassos, 1913

Heterakis numidae Leiper, 1908

Alectoris, Guttera

Yamaguti (1961), Africa

Guineafowl

Yamaguti (1961), Puerto Rico

Bwangamoi (1968), Uganda

Numida meleagris

Graber (1959), Chad

Yamaguti (1961), Africa, the White Nile

Myers et al. (1960), Sudan

Fabiyi (1972), Nigeria

Hodasi (1976), Ghana

Vercruysse et al. (1985), Burkina Faso

Verster \& Ptasinska-Kloryga (1987), South Africa

The White Nile rises from Lake Victoria in Uganda and enters the Sudan where it joins the Blue Nile in Karthoum to form the Nile. White Nile is one of the states of Sudan. 
6. Ascaridia perspicillum (Rudolphi, 1803) Ascaris perspicillum Rudolphi, 1803

Anas acuta, domestic chicken, Meleagris gallopavo, Numida meleagris, Pavo cristatus, Tetrao urogallus, Tetrastes bonasia rupestris, Turdus viscivorus Yamaguti (1961), Europe, Hawaii, India, Indonesia, Japan, Malaya

\section{Superfamily Subuluroidea}

Family SUBULURIDAE (Travassos, 1914) Yorke \& Maplestone, 1926

Subfamily Subulurinae Travassos, 1914

Genus Subulura Molin, 1860

Allodapa auct.

Type species: Subulura acutissima Molin, 1860

1. Subulura acuticauda (Von Linstow, 1901) Railliet \& Henry, 1914

Oxysoma acuticauda Von Linstow, 1901; Heterakis acuticauda (Von Linstow, 1901) Von Linstow, 1909

Numida meleagris

Von Linstow (1901), Kenya

Yamaguti (1961), Africa

2. Subulura brumpti (Lopez-Neyra, 1922)

Allodapa brumpti Lopez-Neyra, 1922

Alectoris graeca, Anas, Colinus virginianus texanus, domestic chicken, Meleagris gallopavo, Numida, Perdix perdix, Streptopelia orientalis

Yamaguti (1961), Europe, Palestine, Cyprus, Cuba, Puerto Rico, Panama, North America, Africa, China

Domestic chicken

Hodasi (1969), Ghana

Mukaratirwa, Hove, Esmann, Hoj, Permin \& Nansen (2001), Zimbabwe

Numida meleagris

Graber (1959), Chad

Hodasi (1976), Ghana

Nfor et al. (1999), Nigeria

3. Subulura dentigera Ortlepp, 1937

Numida meleagris

Ortlepp (1937), South Africa

4. Subulura differens (Sonsino, 1890)

Heterakis differens Sonsino, 1890

Alectoris graeca, Centropus phasianus, domestic chicken, Euplectes orix, Numida meleagris, Perdix perdix canescens, Pternistis bicalcaratus

Yamaguti (1961), Cosmopolitan

5. Subulura suctoria (Molin, 1860)

Heterakis suctoria Molin, 1860; Ascaris forcipata Rudolphi, 1819 , in part

Caprimulgus, Podager, Nyctibius

Yamaguti (1961), Brazil

Burhinus, Coturnix, Numida, Pternistis

Yamaguti (1961), South Africa (Transvaal)
Coturnix coturnix, Lagopus lagopus, Phasianus colchicus, Phasianus colchicus mongolicus, Phasianus colchicus principalis

Yamaguti (1961), Russia, Turkestan

Domestic chicken

Permin et al. (1997), Tanzania

Permin et al. (2002), Zimbabwe

Guttera edouardi

Junker \& Boomker (2007b), South Africa

Numida meleagris

Ortlepp (1937), South Africa

Fabiyi (1972), Nigeria

Vercruysse et al. (1985), Burkina Faso

6. Subulura strongylina (Rudolphi, 1819)

Ascaris strongylina Rudolphi, 1819; Strongylus spiculatus Cobbold, 1861 (Boughton 1939, cited in Yamaguti 1961)

Bonasa, Bucco, Callipepla, Caprimulgus, Chelidoptera, Colinus, Cuculus, Gallus, Malocoptila, Monasa, Nonnula, Numida meleagris, Odontophorus, Perdix, Podager, Tetrao, Tympanuchus

Yamaguti (1961), North America, Puerto Rico

Crypturellus, Odontophorus capueira, Tinamus

Yamaguti (1961), Brazil

Domestic chicken

Permin et al. (1997), Tanzania

Poulsen et al. (2000), Ghana

\section{Order Spirurida Diesing, 1861}

Superfamily Thelazioidea

\section{Family THELAZIIDAE Skrjabin, 1915}

\section{Genus OXYsPiRURA Drasche IN STOSSICH, 1897}

Cramispirura Skrjabin, 1931

Type species: Oxyspirura cephaloptera (Molin, 1860)

1. Oxyspirura mansoni (Cobbold, 1879)

Filaria mansoni Cobbold, 1879; Spiroptera emmerezii Emmerez \& Mégnin, 1901 (Marotel \& Carougeau 1902, cited in Yamaguti 1961)

Domestic chicken, Gallus gallus, Meleagris gallopavo, Pavo cristatus

Yamaguti (1961), Atlantic and Pacific islands, Australia, Democratic Republic of Congo, Formosa, India, Japan, North America

Numida meleagris

Hodasi (1976), Ghana

\section{Superfamily Spiruroidea}

\section{Family GONGYLONEMATIDAE (Hall, 1916, subfam.)}

Sobolev, 1949

\section{Genus Gongylonema Molin, 1857}

Type species: Gongylonema musculi (Rudolphi, 1819) Neumann, 1894

1. Gongylonema ingluvicola Ransom, 1904

Gongylonema sumani Bahlerao, 1933 (Baylis 1939, cited in Yamaguti 1961) 
Gallus, Meleagris, pheasants Yamaguti (1961), Cosmopolitan

Domestic chicken Poulsen et al. (2000), Ghana Magwisha et al. (2002), Tanzania Permin et al. (2002), Zimbabwe

Numida meleagris Nfor et al. (1999), Nigeria

2. Gongylonema congolense Fain, 1955

Cairina moschata Fain (1955), Democratic Republic of the Congo

Gallus Fain (1955), Burundi, Democratic Republic of the Congo, Rwanda

Guttera edouardi Junker \& Boomker (2007b), South Africa

Numida meleagris Fain (1955), Burundi, Democratic Republic of the Congo, Rwanda Fabiyi (1972), Nigeria Graber (1976), Ethiopia Hodasi (1976), Ghana Vercruysse et al. (1985), Burkina Faso Junker \& Boomker (2007b), South Africa

Scleroptila levaillantii Fain (1955), Rwanda

3. Gongylonema sumani Bhalerao, 1933

Gallus gallus

Bhalerao (1933), India

Numida meleagris

Fain \& Thienpont (1958), Burundi

\section{Superfamily Habronematoidea}

Family HABRONEMATIDAE (Chitwood \& Wehr, 1932) Ivaschkin, 1961

Subfamily Habronematinae Chitwood \& Wehr, 1932

\section{Genus Cyrnea Seurat, 1914}

Seurocyrnea Strand, 1929; Skrjabinochona Guschkanskaja, 1931; Chenspirura Hsü, 1957 nec Kou, 1958

Type species: Cyrnea eurycerca Seurat, 1914

1. Cyrnea eurycerca Seurat, 1914

Alectoris, Coturnix, Francolinus, Phasianus, Merops Yamaguti (1961), Africa, Europe

Numida meleagris Ortlepp (1938a), Southern Africa

Alectoris rufa ("Perdix rouge" in Yamaguti [1961]) Yamaguti (1961), Corsica

2. Cyrnea parroti Seurat, 1917

Cyrnea seurati Lopéz-Neyra, 1918; Habronema numidae Ortlepp, 1938; Cyrnea numidae (Ortlepp, 1938)

Alectoris barbara

Yamaguti (1961), Algeria

Alectoris rufa
Yamaguti (1961), Spain

Numida meleagris

Ortlepp (1938b), Malawi, South Africa, Swaziland Fabiyi (1972), Nigeria

Vercruysse et al. (1985), Burkina Faso

Chabaud (1958) divided the genus Cyrnea into the two subgenera Procyrnea Chabaud, 1958 and Cyrnea Chabaud, 1958, subsequently raising them to genus level (Chabaud, 1975). He also synonymized Cyrnea (Cyrnea) numidae Ortlepp, 1938 and Cyrnea (Cyrnea) seurati Lopéz-Neyra, 1918 with Cyrnea (Cyrnea) parroti Seurat, 1917. Our specimens of Cyrnea parroti collected from Numida meleagris in South Africa comply with Ortlepp's (1938b) description of $C$. numidae, but the arrangement of cephalic structures in apical view is that of $C$. parroti. Not having examined Otlepp's (1938b) specimens we adopt the classification of Chabaud (1958) and list Ortlepp's specimens as $C$. parroti.

\section{Genus Sicarius LI, 1934}

Type species: Sicarius dipterum (Popova, 1927) Li, 1934

1. Sicarius caudatus Quentin \& Wertheim, 1975

Numida meleagris Junker \& Boomker (2007b), South Africa

Pycnonotus capensis Quentin \& Wertheim (1975), Israel

Quentin \& Wertheim (1975) described S. caudatus from $P$. capensis present in the collection of the "Helminthological Laboratory Jerusalem" and list Jerusalem as locality. It should be noted that $P$. capensis is endemic to South Africa (Lepage 2007). We therefore conclude that the authors were either looking at birds kept in captivity in Israel, making it difficult to determine the geographic origin of the parasites or did not have any information on the original locality if the birds had been collected in South Africa.

2. Sicarius renatae Cancrini, Balbo \& Iori, 1991

Acryllium vulturinum

Cancrini, Balbo \& Iori (1991), Somalia

\section{Subfamily Histiocephalinae Gendre, 1922}

\section{Genus Hadjelia Seurat, 1916}

Gilsonia Gedoelst, 1919; Stellobronema Guschanskaja, 1937; Sobolevicephalus Parukhin, 1964

1. Hadjelia truncata (Creplin, 1825)

Spiroptera truncata Creplin, 1825; Hadjelia inermis (Gedoelst, 1919)

Aceros corrugatus

Ortlepp (1964), Malucca Islands, Indonesia

Columba livia

Tadros \& Iskander (1975), Egypt

Guttera edouardi, Numida meleagris Junker \& Boomker (2007b), South Africa

Tockus erythrorhynchus, Tockus leucomelas Ortlepp (1964), South Africa

Tockus fasciatus semifasciatus Cram (1927, cited in Ortlepp 1964), Africa 
Coracias benghalensis, Halcyon smyrnensis, Upupa epops

Singh (1949), India

Chabaud \& Campana (1950) synonymized $H$. inermis with $H$. truncata. Ortlepp (1964) did not follow this and recorded his specimens as $H$. inermis. Tadros \& Iskander (1975) synonymized $H$. inermis, $H$. parva and $H$. Ihuillieri with $H$. truncata, designating $H$. truncata as the new type species of the genus.

\section{Family TETRAMERIDAE Travassos, 1914 \\ Subfamily Tetramerinae Railliet, 1915 \\ Genus Tetrameres Creplin, 1846}

Tropisurus Diesing, 1835; Tropidurus Wiegmann, 1835, preoccupied; Gynaecophila Gubanov, 1950; Petrowimeres Tschertkova, 1953; Microtetrameres auct.

Type species: Tetrameres paradoxa (Diesing, 1835)

1. Tetrameres fissispina Diesing, 1861

Acanthophorus horridus Von Linstow, 1876; Acanthophorus tenuis Von Linstow, 1876; Filaria pulicis Von Linstow, 1894

Alectoris, Anas acuta, Anas clypeata, Anas platyrhynchos, Anas querquedula, Aythya ferina, Bucephala clangula, Columba livia, Cygnus melanocoryphus, Fulica atra, Gallus, Melanitta fusca, Meleagris, Meleagris gallopavo, Mergus merganser, Nycticorax nycticorax, Perdix, Somateria molissima, Tachybaptus fluviatilis Yamaguti (1961), Africa, Canton, Europe, Formosa, Guam, India, Malaya, North and South America, Philippines, Russian Turkestan, Siberia, Turkey

Domestic chicken

Le Roux (1926), South Africa

Poulsen et al. (2000), Ghana

Magwisha et al. (2002), Tanzania

Guineafowl

Le Roux (1926), South Africa

Numida meleagris

Fabiyi (1972), Nigeria

Hodasi (1976), Ghana

Vercruysse et al. (1985), Burkina Faso

2. Tetrameres numida Junker \& Boomker 2007

Numida meleagris

Junker \& Boomker (2007a), South Africa

Chabaud (1975) divided the genus Tetrameres into the two subgenera Tetrameres (Tetrameres) Creplin, 1846 and Tetrameres (Microtetrameres) Travassos, 1915. We adopt the view of Anderson (1992) and consider the two as valid genera.

\section{Superfamily Acuarioidea}

Family Acuariidae (Railliet, Henry \& Sisoff, 1912, subfam.)

Subfamily Acuariinae Railliet, Henry \& Sisoff, 1912

\section{Genus Acuaria Bremser, 1811}

Cheilospirura auct.

Type species: Acuaria anthuris (Rudolphi, 1819)
1. Acuaria hamulosa (Diesing, 1851)

Spiroptera hamulosa Diesing, 1851; Cheilospirura hamulosa Diesing, 1861; Spiroptera perforans Centoscudi, 1911

Coturnix coturnix, Gallus gallus, Meleagris, pheasant Yamaguti (1961), Cosmopolitan

Domestic chicken Le Roux (1926), South Africa

Poulsen et al. (2000), Ghana Magwisha et al. (2002), Tanzania

Numida meleagris

Fabiyi (1972), Nigeria

Hodasi (1976), Ghana

\section{Genus Synhimantus Railliet, HenRy \& Sisoff,} 1912

Type species: Synhimantus laticeps (Rudolphi, 1819)

1. Synhimantus spiralis (Linstow, 1883)

Dispharagus spiralis Linstow, 1883

Accipiter, Alectoris, Bonasa, Ciconia, Colinus, Columba, Coracias, Corvus, Gallus, Meleagris, Metopidius, Numida meleagris, Passer, Perdix, Phasianus, Quiscalus, Turdus, Turdus migratorius

Yamaguti (1961), Cosmopolitan

Numida meleagris

Fabiyi (1972), Nigeria

Hodasi (1976), Ghana

Vercruysse et al. (1985), Burkina Faso

\section{Genus Dispharynx Railliet, Henry \& Sisoff,} 1912

Type species: Dispharynx nasuta (Rudolphi, 1819)

1. Dispharynx nasuta (Rudolphi, 1819)

Spiroptera nasuta Rudolphi, 1819

Passer domesticus Yamaguti (1961), Europe

Gallus gallus Yamaguti (1961), Africa, America, Australia, CeyIon, Cuba, Formosa

Domestic chicken, turkeys Gibbons, Jones \& Khalil (1996), no geographic data given

Numida meleagris

Verster \& Ptasinska-Kloryga (1987), South Africa

\section{HOST/PARASITE CHECK LIST}

\section{Order Tinamiformes}

Family Tinamidae (Tinamous)

Genus tinamus Hermann, 1783

Subulura strongylina

Genus Crypturellus Brabourne \& Chubb, 1914

Crypturus

Subulura strongylina 


\section{Order Galliformes}

Domestic chicken

Mediorhynchus gallinarum

Postharmostomum gallinum

Choanotaenia infundibulum

Davainea proglottina

Echinolepis carioca

Hymenolepis cantaniana

Raillietina cohni

Raillietina echinobothrida

Raillietina tetragona

Skrjabinia cesticillus

Acuaria hamulosa

Aonchotheca caudinflata

Ascaridia galli

Ascaridia lineata

Ascaridia perspicillum

Capillaria anatis

Dispharynx nasuta

Eucoleus annulatus

Gongylonema ingluvicola

Heterakis brevispiculum

Heterakis dispar

Heterakis gallinarum

Oxyspirura mansoni

Subulura brumpti

Subulura differens

Subululra strongylina

Subulura suctoria

Syngamus trachea

Tetrameres fissispina

Gallinaceaous birds, galliformes

Mediorhynchus gallinarum

Syngamus trachea

\section{Family Numididae (Guineafowls)}

Crested guineafowl

Postharmostomum gallinum

Guineafowl

Cotugnia crassa

Cotugnia digonopora

Numidella numida

Octopetalum numida

Porogynia paronai

Ascaridia galli

Ascaridia numidae

Tetrameres fissispina

\section{Genus Numida Linnaeus, 1766}

1. Numida meleagris (Linnaeus, 1758) (Helmeted Guineafowl)

Phasianus meleagris

Numida meleagris galeatus Pallas, 1767

Numida galeata

Numida meleagris meleagris (Linnaeus, 1758)

Numida ptilorhyncha
Numida meleagris mitratus Pallas, 1767

Numida mitrata

Numida meleagris marungensis Schalow, 1884

Numida frommi, Numida marungensis, Numida meleagris bodalyae, Numida meleagris frommi, Numida meleagris maxima, Numida meleagris rikwae, Numida mitrata frommi, Numida mitrata maximia, Numida mitrata rikwae, Numida rikwae

Mediorhynchus empodius

Mediorhynchus gallinarum

Mediorhynchus numidae

Mediorhynchus selengensis

Mediorhynchus taeniatus

Dicrocoelium macrostomum

Lutztrema sp.

Postharmostomum gallinum

Abuladzugnia gutterae

Abuladzugnia transvaalensis

Choanotaenia infundibulum

Cotugnia crassa

Cotugnia digonopora

Cotugnia meleagridis

Cotugnia tuliensis

Davainea nana

Davainea paucisegmentata

Davainea paucisegmentata var. dahomeensis

Davainea proglottina

Echinolepis carioca

Hispaniolepis falsata

Hispaniolepis fedtschenkoi

Hispaniolepis hilmyi

Hispaniolepis villosa

Hymenolepis cantaniana

Idiogenes sp.

Metroliasthes lucida

Numidella numida

Octopetalum gutterae

Octopetalum numida

Ortleppolepis multiuncinata

Porogynia paronai

Raillietina angusta

Raillietina cohni

Raillietina echinobothrida

Raillietina pintneri

Raillietina steinhardti

Raillietina tetragona

Raillietina tetragonoides

Raillietina toyohashiensis

Skrjabinia cesticillus

Skrjabinia deweti

Acuaria hamulosa

Ascaridia calcarata

Ascaridia compar

Ascaridia galli

Ascaridia lineata

Ascaridia numidae

Ascaridia perspicillum

Aonchotheca caudinflata

Capillaria anatis

Cyrnea eurycerca 
Cyrnea parroti

Eucoleus annulatus

Gongylonema congolense

Gongylonema ingluvicola

Gongylonema sumani

Hadjelia truncata

Heterakis brevispiculum

Heterakis dispar

Heterakis gallinarum

Heterakis vesicularis

Oxyspirura mansoni

Sicarius caudatus

Sicarius renatae

Subulura acuticauda

Subulura brumpti

Subulura dentigera

Subulura differens

Subulura strongylina

Subulura suctoria

Strongyloides avium

Syngamus trachea

Dispharynx nasuta

Synhimantus spiralis

Tetrameres fissispina

Tetrameres numida

Genus: GutTera WAGLER, 1832

Numidella numida

Raillietina pintneri

Ascaridia numidae

1. Guttera edouardi (Hartlaub, 1867) (Crested Guineafowl)

Numida edouardi (Hartlaub, 1867); Guttera pucherani edouardi (Hartlaub, 1867)

Mediorhynchus taeniatus

Abuladzugnia gutterae

Davainea nana

Metroliasthes lucida

Octopetalum gutterae

Octopetalum numida

Ortleppolepis multiuncinata

Porogynia paronai

Raillietina pintneri

Raillietina steinhardti

Raillietina tetragona

Gongylonema congolense

Hadjelia truncata

Subulura suctoria

\section{Genus: ACRYLliUm Gray, 1840}

Heterakis gallinarum

1. Acryllium vulturinum Gray, 1840 (Vulturine Guineafowl)

Cotugnia shohoi

Raillietina somalensis

Heterakis tenuicauda

Sicarius renatae

\section{Family ODONTOPHORIDAE (New World quails)}

GENUS OREORTYX BAIRD, 1858

Ortyx in Yamaguti (1961)

1. Oreortyx pictus (Douglas, 1829) (Mountain Quail)

Ortyx picta

Ascaridia compar

Heterakis vesicularis

Genus Callipepla Wagler, 1832

Lophortyx

Subulura strongylina

\section{Genus Colinus Goldfuss, 1820}

Ascaridia galli

Eucoleus annulatus

Heterakis vesicularis

Heterakis gallinarum

Subulura strongylina

Synhimantus spiralis

1. Colinus virginianus (Linnaeus, 1758) (Northern Bobwhite)

Tetrao virginianus

Echinolepis carioca

Hymenolepis cantaniana

Skrjabinia cesticillus

1a. Colinus virginianus texanus Lawrence, 1853

Subulura brumpti

Genus Odontophorus Vieillot, 1816

Subulura strongylina

1. Odontophorus capueira (Spix, 1825) (Spot-winged Wood-quail)

Perdix capueira

Subulura strongylina

Family PHASIANIDAE (Partridges, francolins, spurfowls, pheasants, etc.)

Partridge

Ascaridia lineata

Pheasant

Acuaria hamulosa

Gongylonema ingluvicola

Turkey

Dispharynx nasuta

\section{Genus Meleagris Linnaeus, 1758}

Acuaria hamulosa

Ascaridia galli

Ascaridia lineata

Eucoleus annulatus

Gongylonema ingluvicola

Heterakis vesicularis

Heterakis gallinarum

Synhimantus spiralis 
Tetrameres fissispina

1. Meleagris gallopavo Linnaeus, 1758 (Wild Turkey, Common Turkey)

Echinolepis carioca

Hymenolepis cantaniana

Metroliasthes lucida

Numidella numida

Raillietina echinobothrida

Raillietina tetragona

Skrjabinia cesticillus

Ascaridia perspicillum

Oxyspirura mansoni

Subulura brumpti

Tetrameres fissispina

2. Meleagris ocellata Cuvier, 1820 (Ocellated Turkey) Agriocharis ocellata

Ascaridia galli

\section{Genus Bonasa Stephens, 1819}

Ascaridia galli

Ascaridia lineata

Eucoleus annulatus

Heterakis gallinarum

Subulura strongylina

Synhimantus spiralis

1. Bonasa umbellus (Linnaeus, 1766) (Ruffed Grouse)

Tetrao umbellus

Davainea proglottina

Echinolepis carioca

\section{Genus Tetrastes Keyserling \& Blasius, 1840}

Ascaridia compar

1. Tetrastes bonasia (Linnaeus, 1758) (Hazel Grouse)

Bonasa bonasia, Bonasia bonasia, Tetrao bonasia

Hispaniolepis fedtschenkoi

Hymenolepis cantaniana

Skrjabinia cesticillus

1a. Tetrastes bonasia rupestris (Brehm, 1831)

Ascaridia perspicillum

\section{Genus Tetrao Linnaeus, 1758}

Aonchotheca caudinflata

Ascaridia compar

Ascaridia galli

Eucoleus annulatus

Heterakis vesicularis

Heterakis gallinarum

Subulura strongylina

1. Tetrao urogallus Linnaeus, 1758 (Western Capercaillie)

Tetrao major

Skrjabinia cesticillus

Ascaridia perspicillum

2. Tetrao parvirostris Bonaparte, 1856 (Black-billed Capercaillie)
Tetrao urogalloides

Hymenolepis cantaniana

Genus LyRURus Swainson, 1832

Aonchotheca caudinflata

Ascaridia compar

Ascaridia galli

Capillaria anatis

Eucoleus annulatus

Heterakis gallinarum

1. Lyrurus tetrix (Linnaeus, 1758) (Black Grouse)

Tetrao tetrix

Hispaniolepis fedtschenkoi

Skrjabinia cesticillus

\section{Genus Tympanuchus Gloger, 1841}

Cupidonia

Ascaridia lineata

Heterakis gallinarum

1. Tympanuchus phasianellus (Linnaeus, 1758) (Sharptailed Grouse)

Pedioecetes phasianellus (Linnaeus, 1758), Tetrao phasianellus

Ascaridia galli

Ascaridia lineata

Heterakis gallinarum

Subulura strongylina

Genus LAGopus Brisson, 1760

Aonchotheca caudinflata

Heterakis gallinarum

Heterakis vesicularis

1. Lagopus lagopus (Linnaeus, 1758) (Willow Ptarmigan)

Tetrao lagopus

Raillietina tetragona

Skrjabinia cesticillus

Subulura suctoria

1a. Lagopus lagopus scotica (Latham, 1787)

Lagopus scotica

Skrjabinia cesticillus

2. Lagopus muta (Montin, 1781) (Rock Ptarmigan) Raillietina tetragona

\section{Genus Tetragallus Gray, 1832}

1. Tetraogallus caucasicus (Pallas, 1811) (Caucasian Snowcock)

Tetrao caucasica

Hispaniolepis fedtschenkoi

2. Tetraogallus himalayensis Gray, 1843 (Himalayan Snowcock)

Megaloperdix nigelli

Hispaniolepis fedtschenkoi

Genus Alectoris Kaup, 1829

Caccabis in Yamaguti (1961) 
Ascaridia compar

Ascaridia galli

Ascaridia numidae

Cyrnea eurycerca

Heterakis dispar

Heterakis gallinarum

Synhimantus spiralis

Tetrameres fissispina

1. Alectoris barbara (Bonnaterre, 1792) (Barbary Partridge)

Caccabis petrosa, Perdix barbara

Cyrnea parroti

2. Alectoris graeca (Meisner, 1804) (Rock Partridge)

Perdix graeca

Davainea proglottina

Echinolepis carioca

Metroliasthes lucida

Heterakis tenuicauda

Subulura brumpti

Subulura differens

2a. Alectoris graeca saxatilis (Bechstein, 1805)

Caccabis saxatilis chukar

Heterakis tenuicauda

Lepage (2007) states that the original Alectoris graeca has been split into four species, namely Alectoris graeca, Alectoris chukar (Gray, 1830), Alectoris philbyi Lowe, 1934 and Alectoris magna (Prjevalski, 1876).

3. Alectoris rufa (Linnaeus, 1758) (Red-legged Partridge) Caccabis rufa, Coturnix rufa, Tetrao rufus

Metroliasthes lucida

Cyrnea eurycerca

Cyrnea parroti

\section{Genus Francolinus Stephens, 1819}

Ascaridia lineata

Cyrnea eurycerca

Heterakis gallinarum

\section{Genus SCleroptila Blyth, 1852}

1. Scleroptila levaillantii (Valenciennes, 1825) (Redwinged Francolin)

Francolinus levaillantii, Perdix levaillantii

Gongylonema congolense

\section{Genus Pternistis Wagler, 1832}

Subulura suctoria

1. Pternistis natalensis (Smith, 1834) (Natal Spurfowl)

Francolinus natalensis, Pternistes natalensis

Porogynia paronai

2. Pternistis bicalcaratus (Linnaeus, 1766) (Doublespurred Spurfowl)

Francolinus bicalcaratus, Tetrao bicalcaratus

Heterakis brevispiculum

Subulura differens

\section{Genus Perdix Brisson, 1760}

Aonchotheca caudinflata

Ascaridia compar

Ascaridia galli

Capillaria anatis

Eucoleus annulatus

Heterakis vesicularis

Heterakis gallinarum

Subulura strongylina

Synhimantus spiralis

Tetrameres fissispina

1. Perdix perdix (Linnaeus, 1758) (Grey Partridge)

Tetrao perdix

Davainea proglottina

Hymenolepis cantaniana

Metroliasthes lucida

Raillietina echinobothrida

Skrjabinia cesticillus

Subulura brumpti

1a. Perdix perdix canescens Buturlin, 1906

Subulura differens

\section{Genus Coturnix BonnaterRe, 1791}

Aonchotheca caudinflata

Ascaridia compar

Cyrnea eurycerca

Heterakis gallinarum

Heterakis vesicularis

Subulura suctoria

1. Coturnix coturnix (Linnaeus, 1758) (Common Quail)

Tetrao coturnix

Dicrocoelium macrostomum

Echinolepis carioca

Hymenolepis cantaniana

Metroliasthes lucida

Skrjabinia cesticillus

Acuaria hamulosa

Subulura suctoria

Genus ITHAGINIS WAGLER, 1832

Ascaridia galli

Genus Tragopan Cuvier, 1829

Ceriornis

Heterakis gallinarum

Genus Lophophorus TeMminCK, 1813

Heterakis gallinarum

Heterakis vesicularis

\section{Genus Gallus Brisson, 1760}

Aonchotheca caudinflata

Ascaridia compar

Ascaridia lineata

Eucoleus annulatus

Gongylonema congolense 
Gongylonema ingluvicola

Heterakis vesicularis

Strongyloides avium

Subulura strongylina

Synhimantus spiralis

Tetrameres fissispina

1. Gallus gallus (Linnaeus, 1758) (Red Junglefowl)

Gallus ferrugineus, Phasianus gallus

Davainea proglottina

Echinolepis carioca

Hispaniolepis fedtschenkoi

Hymenolepis cantaniana

Metroliasthes Iucida

Raillietina cohni

Raillietina echinobothrida

Raillietina tetragona

Skrjabinia cesticillus

Acuaria hamulosa

Dispharynx nasuta

Gongylonema sumani

Oxyspirura mansoni

1a. Gallus gallus bankiva Temminck, 1813

Metroliasthes lucida

Raillietina echinobothrida

\section{Genus Lophura Fleming, 1822}

Gennaeus

Heterakis gallinarum

1. Lophura nycthemera (Linnaeus, 1758) (Silver Pheasant)

Euplocamus nycthemerus, Gennaeus nycthemerus, Phasianus nycthemerus

Heterakis vesicularis

\section{Genus Syrmaticus WagleR, 1832}

Graphophasianus

Eucoleus annulatus

1. Syrmaticus soemmeringii (Temminck, 1830) (Copper Pheasant)

Graphophasianus soemmeringii, Phasianus soemmeringii

Heterakis gallinarum

Genus Phasianus Linnaeus, 1758

Aonchotheca caudinflata

Ascaridia galli

Ascaridia lineata

Capillaria anatis

Cyrnea eurycerca

Eucoleus annulatus

Heterakis gallinarum

Synhimantus spiralis

1. Phasianus colchicus Linnaeus, 1758 (Common Pheasant, Ring-necked Pheasant)

Hymenolepis cantaniana

Raillietina echinobothrida
Skrjabinia cesticillus

Heterakis vesicularis

Subulura suctoria

1a. Phasianus colchicus mongolicus Brandt, 1844

Phasianus mongolicus turkestanicus

Subulura suctoria

1b. Phasianus colchicus principalis Sclater, 1885

Phasianus principalis

Subulura suctoria

Genus Chrysolophus Gray, 1834

Thaumalea

Aonchotheca caudinflata

Eucoleus annulatus

Heterakis gallinarum

\section{Genus Polyplectron Temminck, 1807}

Heterakis vesicularis

Genus Pavo Linnaeus, 1758

Heterakis gallinarum

Heterakis vesicularis

1. Pavo cristatus Linnaeus, 1758 (Indian Peafowl)

Hymenolepis cantaniana

Raillietina tetragona

Ascaridia perspicillum

Oxyspirura mansoni

2. Pavo muticus Linnaeus, 1766 (Green Peafowl)

Raillietina tetragona

\section{Order Anseriformes}

Syngamus trachea

Family ANATIDAE (Ducks, geese and swans)

Duck

Ascaridia galli

Ascaridia lineata

Goose

Ascaridia lineata

Genus ANSER Brisson, 1760

Cotugnia digonopora

Ascaridia lineata

Capillaria anatis

Heterakis dispar

Heterakis gallinarum

1. Anser cygnoides (Linnaeus, 1758) (Swan Goose)

Cygnopsis cygnoides

Heterakis dispar

\section{Genus Branta Scopoli, 1769}

Heterakis dispar

1. Branta bernicla (Linnaeus, 1758) (White-bellied Brant) 
Check list of helminths of guineafowls (Numididae) and host list of parasites

Anas bernicla

"Bernicla"

Heterakis dispar

\section{Genus Cygnus Bechstein, 1803}

1. Cygnus atratus (Latham, 1790) (Australian Black Swan)

Chenopsis atrata

Heterakis vesicularis

2. Cygnus melanocoryphus (Molina, 1782)

Tetrameres fissispina

\section{Genus Chloephaga Eyton, 1838}

Heterakis dispar

Genus TAdorna BoIE, 1822

Todorna

Heterakis dispar

Genus Cairina Fleming, 1822

Ascaridia galli

Heterakis dispar

Heterakis gallinarum

1. Cairina moschata (Linnaeus,1758) (Muscovy Duck) Gongylonema congolense

\section{Genus Anas Linnaeus, 1758}

Ascaridia lineata

Heterakis dispar

Heterakis gallinarum

Heterakis vesicularis

Subulura brumpti

1. Anas platyrhynchos Linnaeus, 1758 (Mallard)

Anas boschas

Tetrameres fissispina

2. Anas clypeata Linnaeus, 1758 (Northern Shoveler)

Anas spathula, Spatula clypeata

Tetrameres fissispina

3. Anas acuta Linnaeus, 1758 (Northern Pintail)

Dafila acuta

Ascaridia perspicillum

Tetrameres fissispina

According to Lepage (2007) $A$. acuta has been split into $A$. acuta and Anas eatoni, but some authors consider $A$. eatoni a subspecies of $A$. acuta. Peterson (1999) lists the two as separate species.

4. Anas querquedula Linnaeus, 1758 (Garganey)

Querquedula querquedula

Capillaria anatis

Tetrameres fissispina

Genus Aythya BoIE, 1822

1. Aythya ferina (Linnaeus, 1758) (Common Pochard)
Anas ferina, Aristonetta ferina, Nyroca ferina

Tetrameres fissispina

Genus Somateria LeAch, 1819

1. Somateria mollissima (Linnaeus, 1758) (Common Eider)

Anas mollissima

Tetrameres fissispina

Genus Melanitta Boie, 1822

Oedemia, Oidemia

Capillaria anatis

1. Melanitta fusca (Linnaeus, 1758) (Velvet Scooter) Anas fusca, Oidemia fusca

Tetrameres fissispina

\section{Genus Clangula Leach, 1819}

1. Clangula hyemalis (Linnaeus, 1758) (Oldsquaw, Longtailed Duck)

Anas hyemalis, Harelda hyemalis, Ereunetes occidentalis

Capillaria anatis

Genus BuCEPHALA BAIRD, 1858

1. Bucephala clangula (Linnaeus, 1758) (Common Goldeneye)

Anas clangula, Clangula clangula, Glaucionetta clangula

Tetrameres fissispina

Genus Mergus Linnaeus, 1758

Merganser

Capillaria anatis

1. Mergus merganser Linnaeus, 1758 (Common Merganser)

Tetrameres fissispina

\section{Order Turniciformes}

\section{Family TURNICIDAE (Buttonquail)}

Genus TuRnix BonnaterRe, 1791

1. Turnix suscitator (Gmelin, 1789) (Barred Buttonquail)

Tetrao suscitator

Hymenolepis cantaniana

\section{Order Piciformes}

Syngamus trachea

Order Galbuliformes

Family BUCCONIDAE (Puffbirds)

Genus Bucco Brisson, 1760

Subulura strongylina

Genus Malocoptila Gray, 1841

Subulura strongylina 


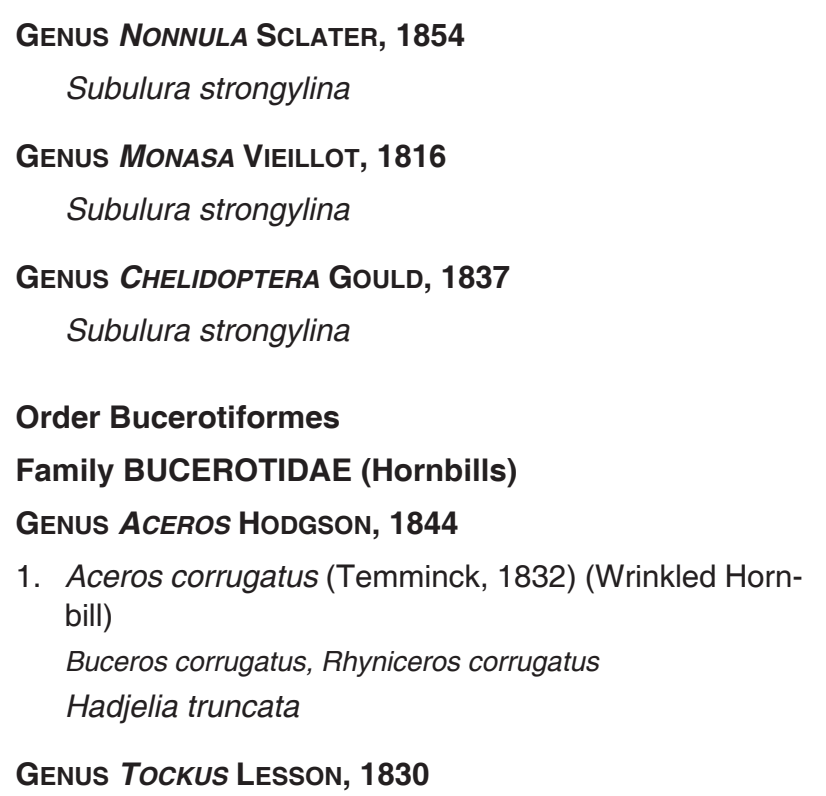

1. Tockus erythrorhynchus (Temminck, 1823) (Red-billed Hornbill)

Buceros erythrorhynchus

Hadjelia truncata

2. Tockus fasciatus semifasciatus (Hartlaub, 1855) (Allied Hornbill)

Lophoceros semifasciatus

Hadjelia truncata

3. Tockus leucomelas (Liechtenstein, 1842) (Southern Yellow-billed Hornbill)

Buceros leucomelas

Hadjelia truncate

Some authors consider T. leucomelas a subspecies of Tockus flavirostris (Rüppell, 1853) (Lepage 2007)

\section{Order Upupiformes}

\section{Family UPUPIDAE (Hoopoes)}

Genus UPUPA LinNAEUS, 1758

1. Upupa epops Linnaeus, 1758 (Hoopoe)

Hadjelia truncata

\section{Family CORACIIDAE (Rollers)}

Genus Coracias Linnaeus, 1758

Synhimantus spiralis

1. Coracias benghalensis (Linnaeus, 1758) (Indian Roller)

Corvus benghalensis

Hadjelia truncata

\section{Genus Halcyon Swainson, 1821}

1. Halcyon smyrnensis (Linnaeus, 1758) (White-throated Kingfisher)

Hadjelia truncata
Family MEROPIDAE (Bee-eaters)

Genus Merops Linnaeus, 1758

Cyrnea eurycerca

Order Cuculiformes

Family CUCULIDAE (Cuckoos, coucals, anis, roadrunners, couas, etc.)

Genus Cuculus Linnaeus, 1758

Subulura strongylina

Genus Centropus Illiger, 1811

1. Centropus phasianinus (Latham, 1802) (Pheasant Coucal)

Cuculus phasianinus

Subulura differens

Order Strigiformes

Family STRIGIDAE (Typical owls)

Genus Strix LinNAEUS, 1758

Heterakis dispar

Heterakis gallinarum

Genus Surnia Dumeril, 1805

Heterakis dispar

Genus Glaucidium Boie, 1826

Heterakis dispar

Family NYCTIBIIDAE (Potoos)

Genus Nyctibius Vieillot, 1816

Subulura suctoria

Family CAPRIMULGIDAE (Nightjars)

Genus Podager Wagler, 1832

Subulura strongylina

Subulura suctoria

Genus Caprimulgus Linnaeus, 1758

Subulura strongylina

Subulura suctoria

Order Columbiformes

Family COLUMBIDAE (Pigeons and doves)

Pigeon

Ascaridia lineata

Genus Columba Linnaeus, 1758

Aonchotheca caudinflata

Synhimantus spiralis

1. Columba livia Gmelin, 1785 (Rock Pigeon) 
Check list of helminths of guineafowls (Numididae) and host list of parasites

Cotugnia digonopora

Raillietina echinobothrida

Hadjelia truncata

Tetrameres fissispina

Genus Streptopelia Bonaparte, 1855

Spilopelia

Ascaridia galli

1. Streptopelia orientalis (Latham, 1790) (Oriental TurtleDove)

Columba orientalis, Turtur orientalis

Subulura brumpti

\section{Order Gruiformes}

Family RALLIDAE (Rails, crakes, moorhens and coots)

\section{Genus Fulica Linnaeus, 1758}

1. Fulica atra Linnaeus, 1758 (Common Coot) Tetrameres fissispina

\section{Family OTIDIDAE (Bustards and korhaans)}

Yamaguti (1961) lists Aonchotheca caudinflata, Heterakis gallinarum and Heterakis vesicularis from Otis without giving the host's species name. It is therefore not clear whether Otis refers to the current genus Otis, Ardeotis, Neotis or Chlamydotis.

Otidiformes in Yamaguti (1961)

Syngamus trachea

\section{Genus Otis Linnaeus, 1758}

1. Otis tarda Linnaeus, 1758 (Great Bustard) Mediorhynchus taeniatus

\section{Genus Ardeotis Le Maout, 1853}

1. Ardeotis arabs (Linnaeus, 1758) (Arabian Bustard) Choriotis arabs, Otis arabs

Mediorhynchus empodius Mediorhynchus taeniatus

\section{Genus Chlamydotis Lesson, 1839}

Houbara

Hetrakis gallinarum

According to Lepage (2007) the common name Houbara has been split into Chlamydotis undulata and Chlamydotis macqueenii.

1. Chlamydotis undulata (Jacquin, 1784) (Houbara Bustard)

Otis houbara, Psophia undulata

Mediorhynchus taeniatus

Hispaniolepis falsata

2. Chlamydotis macqueenii Gray, 1832 (Macqueen's Bustard)

Otis macqueenii

Mediorhynchus taeniatus

\section{Order Charadriiformes}

Family PTEROCLIDIAE (Sandgrouse)

Genus Pterocles Temminck, 1815

Calopterocles

Heterakis gallinarum

1. Pterocles exustus Temminck, 1825 (Chestnut-bellied Sandgrouse)

Pteroclidurus exustus, Pterocles senegalensis

Raillietina cohni

Lepage (2007) lists Pterocles senegalensis as synonym for Pterocles exustus.

2. Pterocles orientalis arenarius (Pallas, 1775) (Eastern Black-bellied Sandgrouse)

Pterocles arenarius

Raillietina cohni

Lepage (2007) and Peterson (1999) list Pterocles arenarius as subspecies of Pterocles orientalis (Linnaeus, 1758).

\section{Family JACANIDAE (Jacanas)}

Genus Metopidius WAGLER, 1832

Synhimantus spiralis

\section{Family BURHINIDAE (Thick-knees)}

\section{Genus BuRhinus ILLiger, 1811}

Oedicnemus

Subulura suctoria

1. Burhinus oedicnemus (Linnaeus, 1758) (Eurasian Thick-knee)

Charadrius oedicnemus, Oedicnemus crepitans

Mediorhynchus taeniatus

\section{Family CHARADRIIDAE}

(Plovers, dotterels, lapwings)

\section{Genus Vanellus Brisson, 1760}

1. Vanellus cinereus (Blyth, 1842) (Grey-headed Lapwing)

Hoplopterus cinereus, Microsarcops cinereus, Pluvianus cinereus

Davainea nana

\section{Order Falconiformes}

Family ACCIPITRIDAE (Eagles, hawks, buzzards, kites, vulures)

Genus ACCIPITER BRISSON, 1760

Synhimantus spiralis

\section{Order Ciconiiformes}

Family PODICIPEDIDAE (Grebes)

Genus TACHYBAPtus ReICHENBACH, 1853

1. Tachybaptus novaehollandiae (Stephens, 1826) (Australian Grebe) 
Podiceps fluviatilis, Podiceps novaehollandiae

Tetrameres fissispina

Family ARDEIDAE (Herons, egrets and bitterns)

"Ardeiformes"

Syngamus trachea

Genus ARdea Linnaeus, 1758

Mediorhynchus empodius

\section{Genus Nycticorax Forster, 1817}

1. Nycticoraxnycticorax (Linnaeus, 1758) (Black-crowned Night-Heron)

Ardea nycticorax

Tetrameres fissispina

Family PELECANIDAE (Pelicans)

"Pelicaniformes"

Syngamus trachea

\section{Family CICONIIDAE (Storks)}

Genus Ciconia Brisson, 1760

Synhimantus spiralis

\section{Order Passeriformes}

Syngamus trachea

\section{Family CORVIDAE (Crows and ravens)}

Genus Corvus Linnaeus, 1758

Heterakis gallinarum

Synhimantus spiralis

\section{Family PYCNONOTIDAE}

Genus Pycnonotus Bole, 1826

1. Pycnonotus capensis (Linnaeus, 1766) (Cape Bulbul) Sicarius caudatus

\section{Family MUSCICAPIDAE (Old World flycatchers)}

Genus Turdus Linnaeus, 1758

\section{Aonchotheca caudinflata Synhimantus spiralis}

1. Turdus viscivorus Linnaeus, 1758 (Mistle Thrush) Ascaridia perspicillum

2. Turdus migratorius Linnaeus, 1766 (American Robin) Planesticus migratorius Synhimantus spiralis

\section{Family STURNIDAE (Starlings)}

\section{Genus Sturnus}

Aonchotheca caudinflata
Family PASSERIDAE (Old World sparrows, sowfinches and relatives)

Genus PASSER Brisson, 1760

Aonchotheca caudinflata

Synhimantus spiralis

1. Passer domesticus (Linnaeus, 1758) (House Sparrow) Fringilla domestica

Dispharynx nasuta

\section{Genus Euplectes Swainson, 1829}

1. Euplectes orix (Linnaeus, 1758) (Red Bishop) Pyromelana oryx Subulura differens

Family FRINGILLIDAE (Canaries and buntings)

Genus Quiscalus Vieillot, 1816

Synhimantus spiralis

\section{ACKNOWLEDGEMENTS}

The first author was supported by a Claude Leon Foundation Postdoctoral Fellowship grant. Funds for this research were made available by the Department of Veterinary Tropical Diseases, as well as the Faculty of Veterinary Science of the University of Pretoria.

\section{REFERENCES}

ANDERSON, R.C. 1992. Nematode parasites of vertebrates, their development and transmission, $1^{\text {st }}$ ed. Wallingford and New York: CAB International.

ANDERSON, R.C., CHABAUD, A.G. \& WILLMOTT, S. (Eds) 1974-1983. CIH keys to the nematode parasites of vertebrates, No. 1-10. Farnham Royal: Commonwealth Agricultural Bureaux.

AYENI, J.S.O., DIPEOLU, O.O. \& OKAEME, A.N. 1983. Parasitic infections of the grey-breasted helmet guinea-fowl (Numida meleagris galeata) in Nigeria. Veterinary Parasitology, 12: 59-63.

BAER, J.G. 1925. Cestodes nouveaux du Sud-Ouest de l'Afrique. Revue Suisse de Zoologie, 31:529-548.

BAER, J.G. 1926. Contributions to the helminth fauna of South Africa. Department of Agriculture. 11 $11^{\text {th }}$ and $12^{\text {th }}$ Reports of the Director of Veterinary Education and Research, Part 1, 61-136.

BAER, J.G. 1933. Contribution á l'étude de la faune helminthologique africaine. Revue Suisse de Zoologie, 40:31-84.

BAER, J.G. 1955. Revision critique de la sous-famille Idiogeninae Fuhrmann 1907 (Cestodes: Davaineidae) et étude analytique de la distribution des espèces. Revue Suisse de Zoologie, 62 (Supplement): 1-51.

BAYLIS, H.A. 1934. Notes on four cestodes. Annals and Magazine of Natural History, 10:587-594.

BELSHAW, R.H.H. 1985. Guinea fowl of the world. Liss, Hampshire: Nimrod Book Services. 
BHALERAO, G.D. 1933. On a new species of Gongylonema (Nematoda) from the domestic fowl. Indian Journal of Veterinary Science and Animal Husbandry, 3:116-119.

BWANGAMOI, O. 1968. Helminth parasites of domestic and wild animals in Uganda. Bulletin of Epizootic Diseases in Africa, 16:429-454.

CANCRINI, G., BALBO, T. \& IORI, A. 1991. Su un nuovo Habronematidae parassita di Acryllium vulturinum: Sicarius renatae sp. n. Parassitologia (Roma), 33:133-136.

CHABAUD, A.G. \& CAMPANA, Y. 1950. Notes sur le genre Hadjelia Seurat, 1916 (Nématodes-Spiruridae). Annales de Parasitologie Humaine et Comparée, 25:435-440.

CHABAUD, A.G. 1958. Essai de classification des nematodes Habronematinae. Annales de Parasitologie, 33:445-508.

CHABAUD, A.G. 1975. CIH keys to the nematode parasites of vertebrates, No. 3. Keys to the genera of the Order Spirurida. Part 2. Spiruroidea, Habronematoidea and Acuaroidea. Farnham Royal: Commonwealth Agricultural Bureaux.

CROWE, T.M. 1977. Variation in intestinal helminth infestation of the helmeted guinea-fowl. South African Journal of Wildlife Research, 7:1-3.

CRUZ E SILVA, J.A. 1971. Contribuição para o estudo dos helmintes parasitas dos vertebrados de Moçambique. Memorias Junta de Investigacoes do Ultramar, 61:1-479.

DEL HOYO, J., ELLIOTT, A. \& SARGATAL, J. (Eds). 1994. Handbook of the birds of the world. Vol. 2. New World vultures to guineafowl. Barcelona: Lynx Edicions.

FABIYI, J.P. 1972. Studies on parasites of the grey-breasted helmet guineafowl (Numida meleagris galeata Pallas) of the Vom area of the Benue Plateau State, Nigeria. I. Helminth parasites. Bulletin of Epizootic Diseases of Africa, 20:235238.

FAIN, A. 1955. Sur un nouveau gongylonème, G. concolense n. sp., parasite de la poule, du canard et des gallinacés sauvages au Congo Belge et au Ruanda-Urundi, Revue de Zoologie et de Botanique Africaines, 51:1-10.

FAIN, A. \& THIENPONT, D. 1958. Notes sur deux Gongylonèmes congolais parasites de l'Oryctérope et de la Pintade. Revue de Zoologie et de Botanique Africaines, 57:113-116.

FATUNMBI, O.O. \& OLUFEMI, B.E. 1982. On the gastro-intestinal helminth parasites of guinea fowl (Numida meleagris galeata Pallas) in Ibadan. African Journal of Ecology, 20:6770.

FUHRMANN, O. 1912. XX. Vogelcestoden. Sitzungsbericht der Kaiserlichen Akademie der Wissenschaften, Wien, 121:181192.

GIBBONS, L.M., JONES, A. \& KHALIL, L.F. 1996. Manual of the 8th international training course on identification of helminths of economic importance. St. Albans: International Institute of Parasitology.

GIBSON, D. (Ed.). 2005. Animal parasitic helminths. Fauna Europaea version 1.2, http://www.Faunaeur.org.

GRABER, M. 1959. Les parasites des animaux domestiques et sauvages de la République du Tchad. Révue d'Élevage et de Médicine Vétérinaire des Pays tropicaux, 12:145-152.

GRABER, M. 1976. Helminthes et helminthiases des animaux domestiques et sauvages d'Ethiopie. Tome 2. 2eme Mission des Vétérinaires français en Ethiopie, Janvier-Mars. '75.

HAZIEV, G.Z. \& KHAN, S.A. 1991. Helminths of guinea fowl (Numida meleagris) in Bashkir ASSR. Veterinary Parasitology, 38:349-353.
HOCKEY, P.A.R., DEAN, W.R.J. \& RYAN, P.G. (Eds). 2005. Roberts-Birds of Southern Africa, VII th edition. Cape Town: The Trustees of the John Voelcker Bird Book Fund.

HODASI, J.K.M. 1969. Comparative studies on the helminth fauna of native and introduced domestic fowls in Ghana. Journal of Helminthology, 43:35-52.

HODASI, J.K.M. 1976. The helminth parasites of the helmet guinea fowl (Numida meleagris galeata Pallas) in Ghana. Bulletin of Animal Health and Production in Africa, 24:8187.

HUDSON, J.R. 1934. Notes on some avian cestodes. Annals and Magazine of Natural History, 14:314-318.

JONES, M.F. 1930. Proceedings of the $121^{\text {st }}$ to $126^{\text {th }}$ Meeting of the Helminthological Society of Washington. Journal of Parasitology, 16:158-169.

JOYEUX, C., BAER, J. \& MARTIN, R. 1936. Sur quelques cestodes de la Somalie-Nord. Bulletin de la Société de Pathologie Exotique, 29:82-96.

JUNKER, K. \& BOOMKER, J. 2006. Mediorhynchus gallinarum (Acanthocephala: Gigantorhynchidae) in Helmeted guineafowls, Numida meleagris, in the Kruger National Park, South Africa. Onderstepoort Journal of Veterinary Research, 73: 283-292.

JUNKER, K. \& BOOMKER, J. 2007a. Tetrameres numida $\mathrm{n}$. sp. (Nematoda: Tetrameridae) from Helmeted guineafowls, Numida meleagris (Linnaeus, 1758), in South Africa. Onderstepoort Journal of Veterinary Research, 74:115-128.

JUNKER, K. \& BOOMKER, J. 2007b. Helminths of guineafowls in Limpopo Province, South Africa. Onderstepoort Journal of Veterinary Research, 74:265-280.

KHALIL, L.F., JONES, A. \& BRAY, R.A. (Eds). 1994. Key to the cestode parasites of vertebrates. Wallingford, UK: CAB International.

KHAN, S.W., KHAN, A.J. \& RAYAZ, S. 1984. Helminth parasites found in the gastro-intestinal tract of guinea fowl, Guttera pucherani, of Peshawar, N.W.F.P., Pakistan. Bulletin of Zoology, University of Peshawar, 2:57-59.

LEPAGE, D. (Ed.). 2007. Avibase. World Wide Web electronic publication. http://www.bsc-eoc.org/avibase/ [4 April 2007].

LE ROUX, P.L. 1926. Helminths collected from the domestic fowl (Gallus domesticus) and the domestic pigeon (Columba livia) in Natal. Department of Agriculture. $11^{\text {th }}$ and $12^{\text {th }}$ Reports of the Director of Veterinary Education and Research, Part 1: 207-217.

LESBOUYRIES, G. 1941. La pathologie des oiseaux. Paris: Vigot Frères.

MAGWISHA, H.B., KASSUKU, A.A., KYVSGAARD, N.C. \& PERMIN, A. 2002. A comparison of the prevalence and burdens of helminth infections in growers and adult free-ranging chickens. Tropical Animal Health and Production, 43:205214.

MEYER, A. 1932. Acanthocephala. Bronns "Klassen und Ordnungen des Tierreichs." Vierter Band, 2. Abteilung, 2. Buch. Lieferung 1, 1-332. Leipzig: Akademische Vertragsgesellschaft.

MYERS, B-J., WOLFGANG, R.W. \& KUNTZ, R.E. 1960. Helminth parasites from vertebrates taken in the Sudan (East Africa). Canadian Journal of Zoology, 38:833-836.

MUKARATIRWA, S., HOVE, T., ESMANN, J.B., HOJ, C.J., PERMIN, A. \& NANSEN, P. 2001. A survey of parasitic nematode infections of chickens in rural Zimbabwe. Onderstepoort Journal of Veterinary Research, 68:183-186. 
NFOR, M.B., AJANUSI, O.J., AGBEDE, R.I.S. \& ESIEVO, K.A.N. 1999. Prevalence of parasites of guinea fowl (Numida meleagris galeata) in Zaria, Nigeria. Bulletin of Animal Health and Production in Africa, 47:103-106.

OOSTHUIZEN, J.H. \& MARKUS, M.B. 1967. The haematozoa of South African Birds. I: Blood and other parasites of two species of game birds. Ibis, 109:115-117.

ORTLEPP, R.J. 1923. The life-history of Syngamus trachealis (Montagu) v. Sieboldt, the gape-worm in chickens. Journal of Helminthology, 1:119-140.

ORTLEPP, R.J. 1937. South African helminths. Part I. Onderstepoort Journal of Veterinary Science and Animal Industry, 9:311-336.

ORTLEPP, R.J. 1938a. South African helminths. Part III. Some mammalian and avian cestodes. Onderstepoort Journal of Veterinary Science and Animal Industry, 11:23-50.

ORTLEPP, R.J. 1938b. South African helminths. Part V. Some avian and mammalian helminths. Onderstepoort Journal of Veterinary Science and Animal Industry, 11:63-104.

ORTLEPP, R.J. 1963. Observations on cestode parasites of guinea-fowl from Southern Africa. Onderstepoort Journal of Veterinary Research, 30:95-118.

ORTLEPP, R.J. 1964. Some helminths recovered from Red- and Yellow-billed Hornbills from the Kruger National Park. Onderstepoort Journal of Veterinary Research, 31:39-52.

PERMIN, A., MAGWISHA, H., KASSUKU, A.A., NANSEN, P., BISGAARD, M., FRANDSEN, F. \& GIBBONS, L.M. 1997. A cross-sectional study of helminths in rural scavenging poultry in Tanzania in relation to season and climate. Journal of Helminthology, 71:233-240.

PERMIN, A., ESMANN, J.B., HOJ, C.H., HOVE, T. \& MUKARATIRWA, S. 2002. Ecto-, endo- and haemoparasites in freeranging chickens in the Goromonzi District in Zimbabwe. Preventive Veterinary Medicine, 54:213-224.

PETERSON, A.P. (Ed.). 1999. Zoological nomenclature resource (Zoonomen). World Wide Web electronic publication. http:// www.zoonomen.net [4 April 2007].

POULSEN, J., PERMIN, A., HINDSBO, O., YELIFARI, L., NANSEN, P. \& BLOCH, P. 2000. Prevalence and distribution of gastro-intestinal helminths and haemoparasites in young scavenging chickens in upper eastern region of Ghana, West Africa. Preventive Veterinary Medicine, 45:237-245.

QUENTIN, J.C. \& WERTHEIM, G. 1975. Helminthes d'oiseaux et de mammifères d'Israël V. - Spirurides nouveaux ou peu connus. Annales de Parasitologie (Paris), 50:63-85.

SAAYMAN, J. 1966. A study of the diet and parasites of Ardeola (Bubulcus) ibis, Numida meleagris and Gallus domesticus from the Eastern Cape Province, South Africa. Ph.D. thesis, University of South Africa.
SANTA CRUZ, A.C.M., ORTIS DE ROTT, M.I. \& RESOAGLI, E.H. 1998. Heterakiosis en Numida meleagris (Aves: Numididae). Boletin chileno de Parasitologia, 53:70-72.

SCHMIDT, G.D. 1986. Handbook of tapeworm identification. Boca Raton, Florida: CRC Press Inc.

SCHMIDT, G.D. \& KUNTZ, R.E. 1977. Revision of Mediorhynchus Van Cleave 1916 (Acanthocephala) with a key to species. Journal of Parasitology, 63:500-507.

SINGH, S.N. 1949. Studies on the helminth parasites of birds in Hyderabad State. Journal of Helminthology, 23:25-38.

SOUTHWELL, T. \& LAKE, F. 1939. On a collection of cestoda from the Belgian Congo. Annals of Tropical Medicine and Parasitology, 33:63-90, 107-123.

TADROS, G. \& ISKANDER, A.R. 1975. Hadjelia truncata (Crepl., 1825) Gendre, 1921 (Spiruridea), a new parasite of pigeons in Egypt and its pathogenicity. Journal of the Egyptian Veterinary Medical Association, 35:283-301.

TALBOT, N.T. 1971. An acanthocephalan parasite, Mediorhynchus gallinarum, of the domesticated fowl in Papua and New Guinea. Australian Veterinary Journal, 47:334-336.

VAN CLEAVE, H.J. 1947. The acanthocephalan genus Mediorhynchus, its history and a review of the species occurring in the United States. Journal of Parasitology, 33:297-315.

VERCRUYSSE, J., HARRIS, E.A., BRAY, R.A., NAGALO, M., PANGUI, M. \& GIBSON, D.I. 1985. A survey of gastrointestinal helminths of the common helmet guinea fowl (Numida meleagris galeata) in Burkina Faso. Avian Diseases, 29:742745.

VERSTER, A. \& PTASINSKA-KLORYGA, Y. 1987. Helminths of helmeted guineafowl in southern Africa. South African Journal of Wildlife Research, Supplement I, 36-38.

VON LINSTOW, O. 1901. Helminthen von den Ufern des NyassaSees. Ein Beitrag zur Helminthenfauna von Süd-Afrika. Jenaer Zeitschrift, 35:409-428.

WOODLAND, W.N.F. 1928. On some new avian cestodes from the Sudan. Parasitology, 20:305-314.

YAMAGUTI, S. 1958. The digenetic trematodes of vertebrates. Vol. I, Part I (Parts IV-V), in Systema Helminthum. New York: Interscience Publishers.

YAMAGUTI, S. 1959. The cestodes of vertebrates. Vol. II, in Systema Helminthum. New York: Interscience Publishers.

YAMAGUTI, S. 1961. The nematodes of vertebrates. Vol. III, Parts I and II, in Systema Helminthum. New York: Interscience Publishers.

YAMAGUTI, S. 1963. Acanthocephala. Vol. V, in Systema Helminthum. New York: Interscience Publishers. 